\title{
Power counting in chiral effective field theory and nuclear binding
}

\author{
C.-J. Yang $\odot,{ }^{1, *}$ A. Ekström, ${ }^{1}$ C. Forssén $\odot,{ }^{1}$ and G. Hagen ${ }^{2,3}$ \\ ${ }^{1}$ Department of Physics, Chalmers University of Technology, SE-412 96 Göteborg, Sweden \\ ${ }^{2}$ Physics Division, Oak Ridge National Laboratory, Oak Ridge, Tennessee 37831, USA \\ ${ }^{3}$ Department of Physics and Astronomy, University of Tennessee, Knoxville, Tennessee 37996, USA
}

(Received 27 November 2020; accepted 27 April 2021; published 10 May 2021)

\begin{abstract}
Chiral effective field theory $(\chi \mathrm{EFT})$, as originally proposed by Weinberg, promises a theoretical connection between low-energy nuclear interactions and quantum chromodynamics (QCD). However, the important property of renormalization-group (RG) invariance is not fulfilled in current implementations and its consequences for predicting atomic nuclei beyond two- and three-nucleon systems has remained unknown. In this work we present a systematic study of recent RG-invariant formulations of $\chi$ EFT and their predictions for the binding energies and other observables of selected nuclear systems with mass numbers up to $A=16$. Specifically, we have carried out $a b$ initio no-core shell-model and coupled cluster calculations of the ground-state energy of ${ }^{3} \mathrm{H},{ }^{3,4} \mathrm{He},{ }^{6} \mathrm{Li}$, and ${ }^{16} \mathrm{O}$ using several recent power-counting (PC) schemes at leading order (LO) and next-to-leading order, where the subleading interactions are treated in perturbation theory. Our calculations indicate that RG-invariant and realistic predictions can be obtained for nuclei with mass number $A \leqslant 4$. We find, however, that ${ }^{16} \mathrm{O}$ is either unbound with respect to the four $\alpha$-particle threshold, or deformed, or both. Similarly, we find that the ${ }^{6} \mathrm{Li}$ ground-state resides above the $\alpha$-deuteron separation threshold. These results are in stark contrast with experimental data and point to either necessary fine-tuning of all relevant counterterms, or that current state-of-the-art RG-invariant PC schemes at LO in $\chi$ EFT lack necessary diagrams-such as three-nucleon forces-to realistically describe nuclei with mass number $A>4$.
\end{abstract}

DOI: 10.1103/PhysRevC.103.054304

\section{INTRODUCTION}

Effective field theory (EFT) [1] provides a theoretical framework for predicting physical phenomena-normally within some energy domain of interest-without knowing or assuming the full details of the underlying physics. Indeed, most physical systems exhibit many characteristic energy and length scales, and with the tools of EFT we can exploit such scale separations for analyzing physical processes. Although it is not always obvious, this approach is used throughout the physical sciences. For instance, not much is gained by including the quark degrees of freedom in the quantum electrodynamic description of the hydrogen atom.

The EFT philosophy appears particularly suitable for application in low-energy nuclear physics calculations. Indeed, computing nuclei directly from the Lagrangian of quantum chromodynamics (QCD), via lattice QCD methods, is extremely complicated, and in most cases computationally

\footnotetext{
*chiehjen@chalmers.se
}

Published by the American Physical Society under the terms of the Creative Commons Attribution 4.0 International license. Further distribution of this work must maintain attribution to the author $(s)$ and the published article's title, journal citation, and DOI. Funded by Bibsam. challenging or intractable, in particular within the nonperturbative region of QCD.

Chiral effective field theory $(\chi \mathrm{EFT})[2-15]$ promises a viable method for deriving the low-energy description of the pion-mediated nuclear interaction that is also constrained by the symmetries of QCD, and in particular the spontaneous breaking of the approximate chiral symmetry of quarks. This approach could potentially connect the description of atomic nuclei to the standard model of particle physics. Furthermore, an EFT offers a handle on estimating the impact of omitted higher-order dynamics that also contribute to the epistemic uncertainty of the approach. If the $\chi$ EFT description of the nuclear interactions complies with all field-theoretical requirements, in particular renormalization group (RG) invariance, it could significantly increase the predictive power of $a b$ initio computations of nuclear properties [16-25]. In this paper we present a first study of the nuclear binding mechanism in selected low-mass nuclei using RG-invariant formulations of the strong nuclear interaction.

The overarching strategy in $\chi$ EFT is to start from an effective Lagrangian including all interaction terms with the same symmetries as QCD below the chiral symmetry-breaking scale $\approx 1 \mathrm{GeV}$. Applying the methods of chiral perturbation theory yields a potential description of the internucleon interaction in terms of irreducible multipion exchanges and zero-range contact interactions. In this sense, $\chi E F T$ is often viewed as a low-energy expansion of QCD, dressed in the relevant degrees of freedom-pions and nucleons-and 
sometimes the lowest excitation of the nucleon, i.e., the $\Delta$ (1232)-isobar [5,26-28].

In EFT studies of nuclei, one aims at predicting low-energy nuclear observables using an order-by-order improvable potential-expansion in terms of a small parameter constructed as a ratio between the physically relevant soft and hard scales. In $\chi$ EFT, the hard momentum-scale is $\Lambda_{b} \approx 0.5-1 \mathrm{GeV}$, and the soft scale is $Q=\max \left(q, m_{\pi}\right)$, where $q$ denotes the external (initial or final state) momentum scale of the interacting nucleons, and $m_{\pi} \approx 140 \mathrm{MeV}$ denotes the pion mass. Up to a certain order $v$ in the chiral expansion, only a finite number of interaction terms, or diagrams, contribute. The organizational scheme for assigning a diagram to a specific order in the EFT according to its expected importance is referred to as power counting (PC). Besides renormalizing the potential, the PC should also ensure RG-invariant amplitudes, i.e., observables. $\mathrm{RG}$-invariance is a crucial requirement in any EFT. When integrating out the pion degree of freedom, one can construct a so-called pionless EFT, which is easier to deal with analytically and for which the path towards renormalizability is clear [29]. This framework has been employed for successfully describing few-nucleon systems, predominantly helium isotopes [30-33], and for extrapolating lattice QCD predictions [34]. However, pionless EFT appears inadequate for predicting realistic properties of light- and medium-mass nuclei heavier than ${ }^{4} \mathrm{He}$ [35-38], and it remains an open question whether subleading orders will provide a remedy.

In nuclei, the likely importance of internucleon interactions with external momenta $q \gtrsim m_{\pi}$ suggests the need for an explicit inclusion of pion physics and the use of $\chi$ EFT. Unfortunately, the presence of the pion propagator typically complicates the Schrödinger equation to the extent that analytical studies become intractable. One must therefore resort to numerical checks of RG-invariance at each chiral order. For nuclear structure calculations-which are always performed within a truncated Hilbert space-enlarging the model space will determine whether all high-momentum (short range) dynamics are properly accounted for as contact interactions. In practice, this is typically done by increasing the imposed momentum cutoff $\Lambda$ that serves to regularize the potential. In this procedure, additional high-momentum details are explicitly exposed. RG invariance is destroyed if the short-range couplings (counterterms) — typically referred to as low-energy constants (LECs) - fail to run with the additional high-momentum ingredients. The resulting lack of $\mathrm{RG}$ invariance yields observable predictions that depend on the regularization procedure. In contrast, an EFT is order-byorder renormalizable if the predicted observables evaluated up to order $v$ have residual cutoff dependence equal to or less than $\left(\frac{Q}{\Lambda}\right)^{v+1}$.

Chiral perturbation theory provides an order-by-order renormalizable framework for constructing a low-energy EFT of QCD and has also been applied quite successfully to the single-nucleon sector with explicit pions, see, e.g., Refs. [39,40]. Problems emerge, however, with the inclusion of two or more nucleons. These difficulties were not entirely clear in the early days of $\chi$ EFT [2] because it was initially assumed that the PC employed in the single-nucleon sector would successfully carry over to renormalize also the mult- inucleon sector. This approach is colloquially referred to as Weinberg power counting (WPC) and is the de facto PC employed in quantitatively realistic descriptions of atomic nuclei. Nevertheless, it is already well known that $\chi$ EFT based on WPC will not generate RG-invariant results for observables, see, e.g., Ref. [41].

Nowadays, there exist several PCs for $\chi$ EFT [42-53] that produce RG-invariant nucleon-nucleon (NN) scattering amplitudes. However, in the present paradigm of $a b$ initio computations, such PCs remain unexplored in studies of atomic nuclei with mass number $A \geqslant 4$. In fact, there are merely two attempts to demonstrate RG invariance of nuclear structure calculations beyond the NN sector. These are Faddeev-type calculations of the three-body systems ${ }^{3} \mathrm{H},{ }^{3} \mathrm{He}$ [41,54].

In this work we significantly broaden the established field of low-energy nuclear theory by applying RG-invariant $\chi$ EFT interactions to selected nuclei with mass numbers $A \leqslant 16$. This constitutes an important leap forward in the exploration of RG-invariant formulations of $\chi$ EFT [44]. We consider some of the most recent RG-invariant $\chi \mathrm{EFT}$ formulations [42-44], and employ the no-core shell model (NCSM) [55,56] and the coupled-cluster (CC) method [21,57-62] to calculate the ground-state energy and nuclear charge radius of ${ }^{3} \mathrm{H},{ }^{3} \mathrm{He}$, ${ }^{4} \mathrm{He},{ }^{6} \mathrm{Li}$, and the ground-state energy of ${ }^{16} \mathrm{O}$, respectively. Note that the total binding energy for the system is the negative of the ground-state energy. The NCSM gives, in principle, an exact solution to the many-nucleon Schrödinger equation, but is limited to light nuclei due to the exponential increase in computing cost with the system size (a combined measure of the number of basis states and nucleons). On the other hand the $\mathrm{CC}$ method has a much softer (polynomial) scaling with the system size, and it gives a controlled and systematically improvable approximation to the exact solution for the wave function $[21,62]$. The access to consistently increasing computational power and the development of similarity RG techniques [18] enables computation of nuclei as heavy as ${ }^{100} \mathrm{Sn}$ using $a b$ initio $\mathrm{CC}$ and similar methods [63-66]. In this work, however, we are focusing on RG invariance and must therefore explore relatively large values for the regulator cutoff in the interactions. In both NCSM and CC, the size of the employed basis must go hand-in-hand with a large cutoff to resolve the short-range part of the interaction while also capturing the long-range part of the wave function. It is therefore a big computational challenge to predict nuclear many-body observables using a nuclear interaction with strong high-momentum details. Where possible, we employ recent extrapolation techniques [67-71] to obtain reasonably converged results for the ground-state energy of the nuclei considered in this work.

In a renormalizable $\chi$ EFT, the subleading contributions, i.e., beyond leading order (LO), are treated in perturbation theory. Indeed, in order to achieve RG invariance at the NN level it has been shown that-due to a Wigner bound-like effect [72,73] — one has to either treat all subleading contributions perturbatively or promote at least two short-range contact terms nonperturbatively at the same time [74-76]. ${ }^{1}$

\footnotetext{
${ }^{1}$ There also exists a renormalization scheme which corresponds to using infinitely many contact terms [77,78].
} 
In this work we will follow the strategy of perturbatively including subleading contributions, and we also demonstrate how a Hellmann-Feynman procedure can be used to achieve this without modifying existing many-body solvers.

This paper is organized as follows: In Sec. II, we briefly introduce the $\chi$ EFTs used in this work and Weinberg's initial approach. Then, in Sec. III, we present ab initio predictions for ${ }^{3} \mathrm{H}$ and ${ }^{3,4} \mathrm{He}$ up to NLO in a well-known RG-invariant PC. Corresponding NCSM and CC calculations for ${ }^{6} \mathrm{Li}$ and ${ }^{16} \mathrm{O}$, respectively, are presented in Sec. IV. In Sec. V we describe some additional and relevant PC schemes based on a dibaryon-field, a separable version of the dibaryon field [45,79], and a perturbative treatment of most $P$ waves [53]. In this section we also present the $\mathrm{CC}$ predictions for the ground-state energy in ${ }^{16} \mathrm{O}$ using such alternative $\mathrm{PC}$ schemes. We summarize our findings and their implications in Sec. VI.

\section{MODIFIED WEINBERG POWER COUNTING}

Detailed properties of several nuclear systems can nowadays be successfully described by solving the nonrelativistic Schrödinger equation using sophisticated potentials based on Weinberg's initial approach [2,3]; see, e.g., Refs. [64,80-90]. Such interactions also enable a description of low-energy NN scattering data with an accuracy comparable to [9] and beyond [91-93] existing high-precision and phenomenological potentials [94-96]. Despite the fact that WPC has enabled successful $a b$ initio models of the strong nuclear interaction, there are several reasons for modifying Weinberg's initial prescription [2,3] for generating NN and three-nucleon (NNN) potentials. In particular, the amplitude produced from Weinberg's prescription for generating the potential is not RG invariant [41,97-99]. We emphasize that WPC is important for guiding experimental and theoretical analyses of nuclei and nuclear systems, but it does not necessarily lead us closer to analyzing nuclei from first principles, i.e., from QCD. We should also point out that there exist arguments $[77,78,100$ 102] for an alternative view on renormalization in $\chi$ EFT. See, e.g., Refs. [15,103-110] for extensive discussions on opposing views regarding this topic. Besides the above problems, Weinberg's prescription also lacks a pion-mass-dependent contact term-which is demanded by RG in the chiral extrapolation applications [111,112]. Although this is an important aspect of the theory, it is not the focus of our present work.

The conventional implementation of $\chi \mathrm{EFT}$ proceeds in two steps: First, one constructs the long-range (pionexchange) potential from the chiral Lagrangian. Then one collects the necessary short-range diagrams into a contact potential to cancel the divergences of the aforementioned long-range pion potential, and subsequently iterates the sum of all potential terms nonperturbatively in the LippmannSchwinger or Schrödinger equation to obtain the amplitudes for constructing, e.g., the scattering $S$ matrix. The resulting potentials are singular at short distances (large momenta), and therefore require regularization using a regulator function $f_{R}$ with an ultraviolet cutoff $\Lambda$. In this work we use a momentum-space representation and employ a standard, non- local, regulator function

$$
f_{R}(p ; \Lambda)=\exp \left[(-p / \Lambda)^{2 n}\right],
$$

with $n=2$. We denote the initial (final) relative momenta with $\mathbf{p}\left(\mathbf{p}^{\prime}\right)$, and use $\mathbf{q}=\mathbf{p}^{\prime}-\mathbf{p}$ for the momentum transfer. Note that local regulators can also be adopted, and this has been explored in coordinate-space quantum Monte Carlo calculations up to NNLO in $\chi$ EFT using WPC [113,114]. One can also mix the local and nonlocal formalism $[11,115]$. See also Ref. [116] for a detailed discussion of some of the observed artifacts induced by different regulator functions.

Clearly, predictions of observables should not depend on the chosen regulator or the value of the regulator cutoff $\Lambda$, i.e., the LECs in the contact potential must act as counterterms and run with $\Lambda$ at each chiral order. To achieve RG-invariant amplitudes we must modify WPC.

\section{A. Leading order}

At LO in WPC, the interaction potential consists of the well-known one-pion-exchange potential (OPE) accompanied by two NN contact terms acting in the singlet and triplet $S$ waves. In momentum space it is represented as

$$
V_{\mathrm{LO}}^{\mathrm{WPC}}\left(\mathbf{p}, \mathbf{p}^{\prime}\right)=\frac{g_{A}^{2}}{4 f_{\pi}^{2}} \tau_{1} \cdot \tau_{2} \frac{\left(\sigma_{1} \cdot \mathbf{q}\right)\left(\sigma_{2} \cdot \mathbf{q}\right)}{m_{\pi}^{2}+\mathbf{q}^{2}}+\tilde{C}_{{ }}{ }_{S_{0}}+\tilde{C}_{{ }^{3} S_{1}} .
$$

Here, $\tilde{C}_{{ }}, \tilde{C}_{{ } S_{1}}$ denote the LO contact LECs acting in separate partial waves. Above, and in the following, we suppress the $\Lambda$ dependence of the LECs. Also, we adopt the value $g_{A}=$ 1.27 for the axial coupling and $f_{\pi}=93 \mathrm{MeV}$ for the piondecay constant, respectively, and employ $m_{\pi}=138 \mathrm{MeV}$ and $m_{N}=938.9 \mathrm{MeV}$ for the pion and nucleon mass, respectively.

It is well established that $V_{\mathrm{LO}}^{\mathrm{WPC}}$ produces nonrenormalizable amplitudes in the singular and attractive partial-wave channels, e.g., ${ }^{3} P_{0}$ and ${ }^{3} P_{2}-{ }^{3} F_{2}$ [41]. A remedy of this situation can be achieved by promoting two additional contact terms at the potential level (otherwise subleading in WPC) to the ${ }^{3} P_{0}$ and ${ }^{3} P_{2}$ channels (one for each), and by treating all partial waves with angular-momentum quantum number $\ell>1^{2}$ perturbatively [53]. These modifications will lead to RG-invariant NN amplitudes at LO. Due to its similarity with WPC at LO, we refer to this RG-invariant PC as modified Weinberg power counting (MWPC) throughout this work. The corresponding momentum-space potential at $\mathrm{LO}$ is given by

$$
V_{\mathrm{LO}}^{\mathrm{MWPC}}\left(\mathbf{p}, \mathbf{p}^{\prime}\right)=V_{\mathrm{LO}}^{\mathrm{WPC}}\left(\mathbf{p}, \mathbf{p}^{\prime}\right)+\left(\tilde{C}_{3 P_{0}}+\tilde{C}_{3 P_{2}}\right) p p^{\prime} .
$$

At this order we obtain the amplitudes nonperturbatively in all partial waves with $\ell \leqslant 1$. Note that MWPC and WPC coincide with each other in $S$ waves. Furthermore, the $S$-wave component of the nuclear interaction has a large impact on nuclear binding energies, and the NN scattering phase shifts from MWPC in the ${ }^{1} S_{0}$ partial wave show a sizable overattraction with respect to the Nijmegen partial-wave analysis

\footnotetext{
${ }^{2}$ In Sec. V C we explore a PC with a perturbative treatment of most $P$ waves.
} 


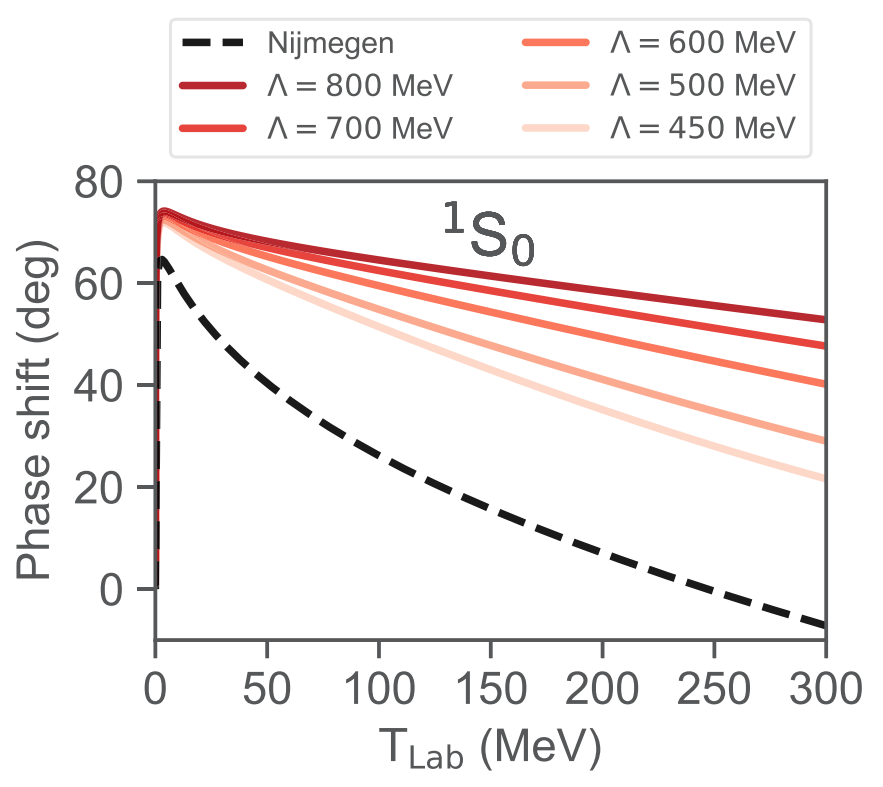

FIG. 1. The ${ }^{1} S_{0} \mathrm{NN}$ scattering phase shifts at LO in MWPC as a function of laboratory scattering energy $T_{\mathrm{Lab}}$ for several values of the regulator cutoff $\Lambda$. Note that the over-attraction persists even for the lowest cutoff value $\Lambda=450 \mathrm{MeV}$.

[96] even at very low scattering energies, see Fig. 1. To remedy this unphysical over-attraction, it is motivated to consider alternative PCs, which will be discussed further in Sec. V. Throughout this work, we neglect any isospin-breaking contributions in the PC schemes we employ. However, we do include the Coulomb interaction $\approx \alpha / r$ ( $\alpha$ is the fine-structure constant) nonperturbatively at $\mathrm{LO}$ in all ab initio calculations although it, in principle, requires special treatment due to a renormalization issue. See, e.g., the discussion in Ref. [117].

For quantitative predictions we must infer numerical values of the relevant LECs for every value of the regulator cutoff $\Lambda$ we employ. The primary goal of this work is to present the first predictions of bulk properties, primarily the groundstate energy, of selected atomic nuclei up to ${ }^{16} \mathrm{O}$ using MWPC, as well as some other RG-invariant NN interactions. Thus, to proceed with a first analysis we straightforwardly determine the numerical values for the contact LECs such that the chosen theory reproduces the experimental values for a selected set of calibration observables. A future procedure could entail a more detailed statistical inference analysis of the underlying EFT uncertainty as well as the LECs themselves [118-120].

Since we employ a pionful theory, we generally prefer to renormalize the LECs at a relative NN momentum $k$ corresponding to $m_{\pi}$ where possible. This relative momentum corresponds approximately to a laboratory scattering energy $T_{\mathrm{Lab}}=40 \mathrm{MeV}$. However, to accommodate the nearly bound character of the ${ }^{1} S_{0}$ channel we had to pick a different kinematical calibration point for this channel. Indeed, matching the only counterterm in this channel to reproduce the phase shift at $k \approx m_{\pi}$ leads to a rather poor reproduction of the phase shift at $k<m_{\pi}$. We therefore fit the LO LEC in the ${ }^{1} S_{0}$ channel to reproduce the $S$-wave scattering length $a_{0}=-23.7$ fm [121]. Also, in the ${ }^{3} S_{1}-{ }^{3} D_{1}$ channel, we renormalize the

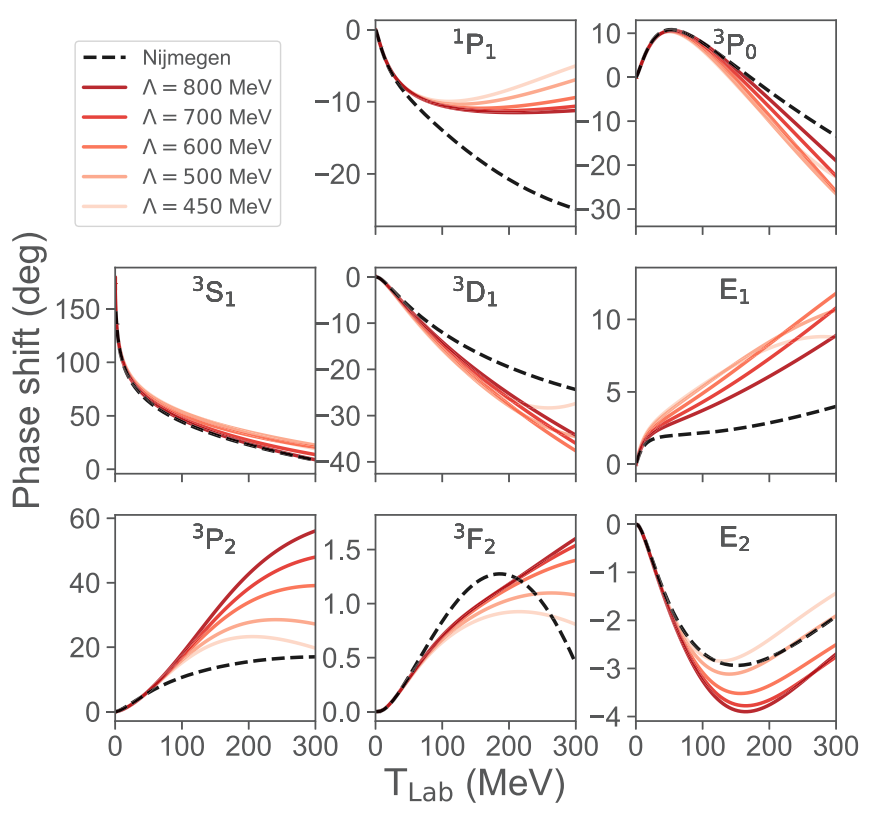

FIG. 2. Selected phase shifts at LO in MWPC as a function of laboratory scattering energy $T_{\mathrm{Lab}}$. Here, the $C_{{ }^{3} S_{1}}$ LEC is fitted to reproduce the deuteron binding energy while the $P$-wave LECs are fit to reproduce the phase shifts at $T_{\mathrm{Lab}}=40 \mathrm{MeV}$.

$\tilde{C}_{3_{S_{1}}}$ counterterm to reproduce the deuteron binding energy. For ${ }^{3} P_{2}-{ }^{3} F_{2}$, when calibrating to reproduce the phase shift at $k \approx m_{\pi}$, we observed a sizable over-attraction with respect to the Nijmegen analysis for $k>m_{\pi}$. This is clearly visible in Fig. 2. To study the impact of this over-attraction, we alternatively fit the ${ }^{3} P_{2}-{ }^{3} F_{2}$ phase shifts at $T_{\text {Lab }}=200 \mathrm{MeV}$. We will refer to these different LO interactions as MWPC(40) and MWPC(200). The latter fit yields a dramatically different result for this coupled channel, see Fig. 3. As expected, and as we will see in Sec. III, this has a negligible, percent-level
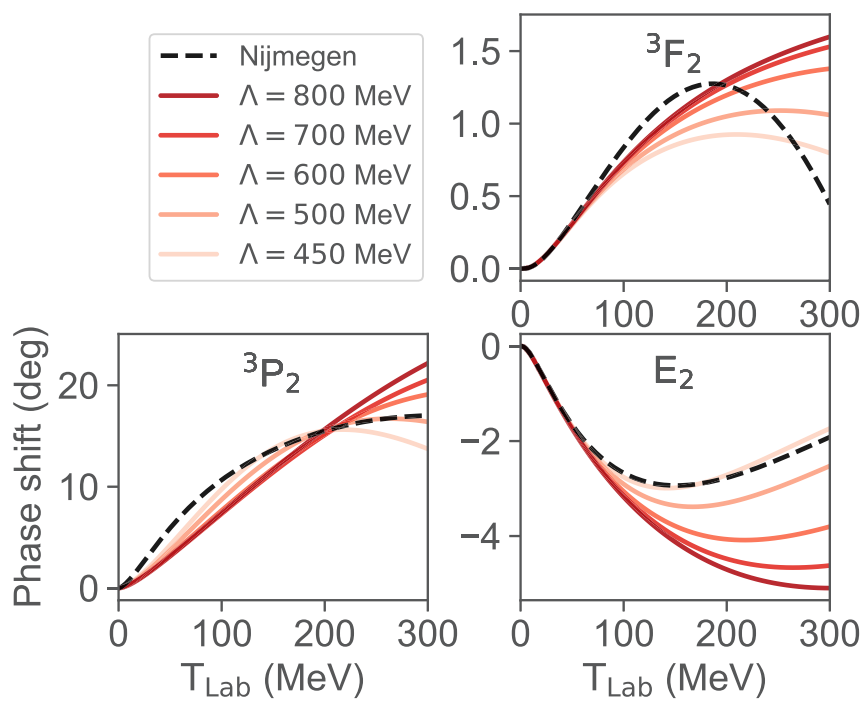

FIG. 3. ${ }^{3} P_{2}-{ }^{3} F_{2}$ phase shift at LO in MWPC as a function of laboratory kinetic energy $T_{\mathrm{Lab}}$. Here the relevant LEC is fitted to reproduce the phase shifts at $T_{\mathrm{Lab}}=200 \mathrm{MeV}$. 
impact on the binding energy in few-nucleon systems. On the other hand, the details of the fitting strategy appears to have a significant effect on the $a b$ initio description of the ground-state energy in ${ }^{16} \mathrm{O}$. This is a key finding of this work, and we will return to this point in more detail in Sec. IV. In MWPC, at the cutoff $\Lambda=750$ (1050) MeV, deep spurious bound states start to appear in the ${ }^{3} P_{0}\left({ }^{3} S_{1}-{ }^{3} D_{1}\right)$ channel. We follow the standard projection method as listed in Appendix B of Ref. [41] to remove those states. Ideally, an associated parameter $\lambda$, that is used to control the projection of the spurious states, should be taken very large. However, this would also result in extremely large values for the matrix elements in the $a b$ initio calculations and will induce numerical problems. We find it sufficient to employ $\lambda \approx 10-15 \mathrm{GeV}$.

\section{B. Next-to-leading order}

According to the analyses in Refs. [42-45,51], the NLO contribution in MWPC has to come one chiral order before the appearance of the two-pion-exchange potential. In fact, the entire NLO contribution to the amplitude only consists of ${ }^{1} S_{0}$ short-range interactions

$$
V_{\mathrm{NLO}}^{\mathrm{MWPC}}\left(\mathbf{p}, \mathbf{p}^{\prime}\right)=C_{{ }^{1} S_{0}}+\hat{C}_{{ }^{S_{0}}}\left(p^{2}+p^{\prime 2}\right) .
$$

We treat subleading orders perturbatively and this ${ }^{1} S_{0}$ contribution is evaluated in the distorted-wave Born approximation. To be clear, there are three differences between the NLO in MWPC and WPC:

(i) NLO in MWPC appears one chiral order earlier than in WPC.

(ii) NLO in MWPC contains only short-range terms as listed in Eq. (4).

(iii) The NLO interaction is treated perturbatively in MWPC, rather than being iterated to all orders as in WPC.

We note that the NLO contribution in Eq. (4) contains two additional LECs at NLO, acting only in the ${ }^{1} S_{0}$ channel. However, the LEC $C_{{ }^{1} S_{0}}$ is the NLO correction to $\tilde{C}_{{ } S_{0}}$. Thus, we effectively only have two LECs in the ${ }^{1} S_{0}$ channel up to, and including, NLO. Still, this gives us plenty of freedom to describe the corresponding phase shift. In this work we choose to renormalize the ${ }^{1} S_{0}$ LEC to reproduce the scattering length $a_{0}=-23.7 \mathrm{fm}$ and the Nijmegen phase shift at $T_{\mathrm{Lab}}=250 \mathrm{MeV}$. As expected, the resulting predictions exhibits a very nice agreement with the Nijmegen analysis, as shown in Fig. 4. Note also the very weak dependence on the regulator cutoff $\Lambda$ at this order (cf. Fig. 1). The LO and NLO predictions for the effective range $r_{0}$, as a function of the regulator cutoff $\Lambda$, are shown in Fig. 5. Here, we employ a larger range of momentum cutoffs just to demonstrate the expected plateau behavior of a RG-invariant amplitude. In NN calculations it is typically not challenging to take $\Lambda$ to even larger values, e.g., 10-20 GeV. However, most ab initio methods for solving the many-body Schrödinger equation fail to converge for $\Lambda \gtrsim 600 \mathrm{MeV}$ due to strong induced wave function correlations, and limitations on the employed modelspace sizes. Several examples of this will be encountered below.

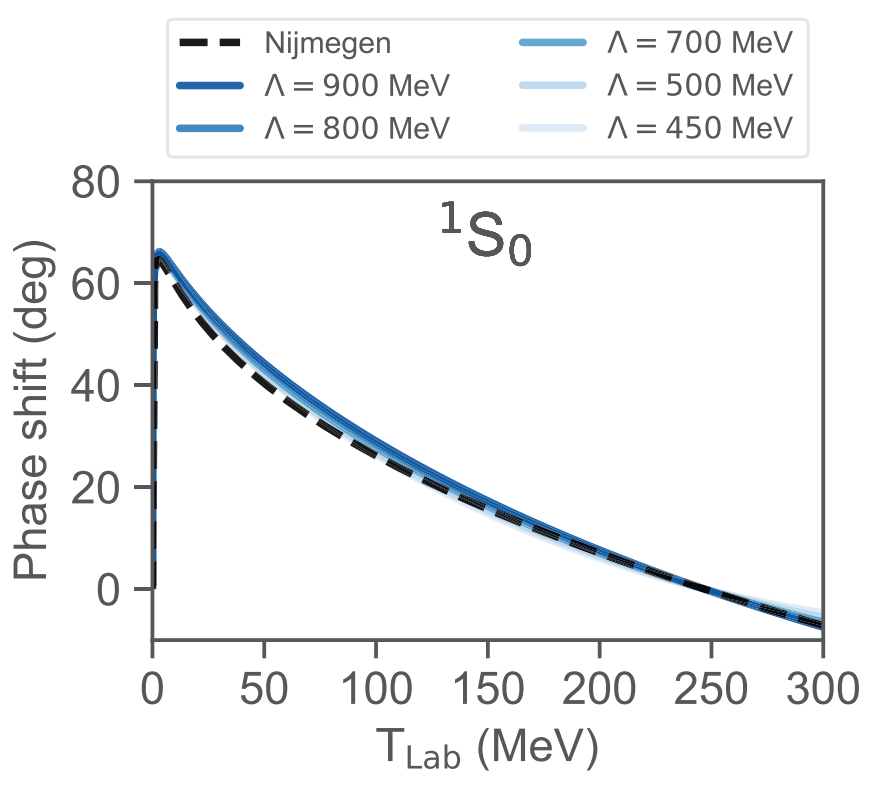

FIG. 4. The ${ }^{1} S_{0}$ phase shift up to NLO in MWPC as a function of laboratory scattering energy $T_{\mathrm{Lab}}$. The relevant LECs are fitted to reproduce the scattering length $a_{0}=-23.7 \mathrm{fm}$ and and the Nijmegen phase shift at $T_{\text {Lab }}=250 \mathrm{MeV}$.

\section{PREDICTIONS FOR ${ }^{3} \mathrm{H}$ AND ${ }^{3,4}$ He USING $\chi$ EFT POTENTIALS IN MODIFIED WEINBERG POWER COUNTING}

In this section we present the results from NCSM fewnucleon calculations of the bulk properties of ${ }^{3} \mathrm{H}$ and ${ }^{3,4} \mathrm{He}$ based on the $\chi$ EFT potentials in MWPC at LO and NLO presented above. For these calculations we employed the MWPC(40) LO potential with the $\tilde{C}_{3 P_{2}}$ LEC calibrated to reproduce the Nijmegen phase shifts at relative momentum $k \sim m_{\pi}\left(T_{\mathrm{Lab}}=40 \mathrm{MeV}\right)$. For comparison we also performed NCSM calculations using the MWPC(200) LO potential where the ${ }^{3} P_{2}-{ }^{3} F_{2}$ channel was renormalized at $T_{\mathrm{Lab}}=$ $200 \mathrm{MeV}$. However, since the two renormalized ${ }^{3} P_{2}-{ }^{3} F_{2}$ partial-wave contributions are both small at lower energies (e.g., $T_{\mathrm{lab}}<100 \mathrm{MeV}$ ), these two different strategies for calibrating the $\tilde{C}_{3 P_{2}}$ LEC produce at most $5 \%$ relative differences in the energies and radii for $A=3,4$ nuclei.

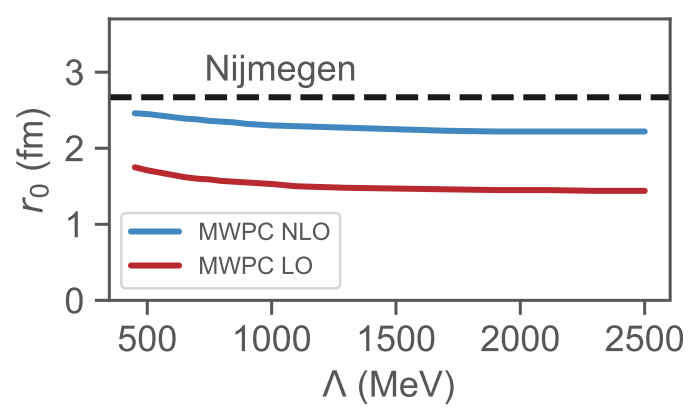

FIG. 5. Predictions for the effective range $r_{0}$ in the ${ }^{1} S_{0}$ channel up to NLO in the MWPC. The MWPC NLO result is closest to the Nijmegen value (dashed line). 

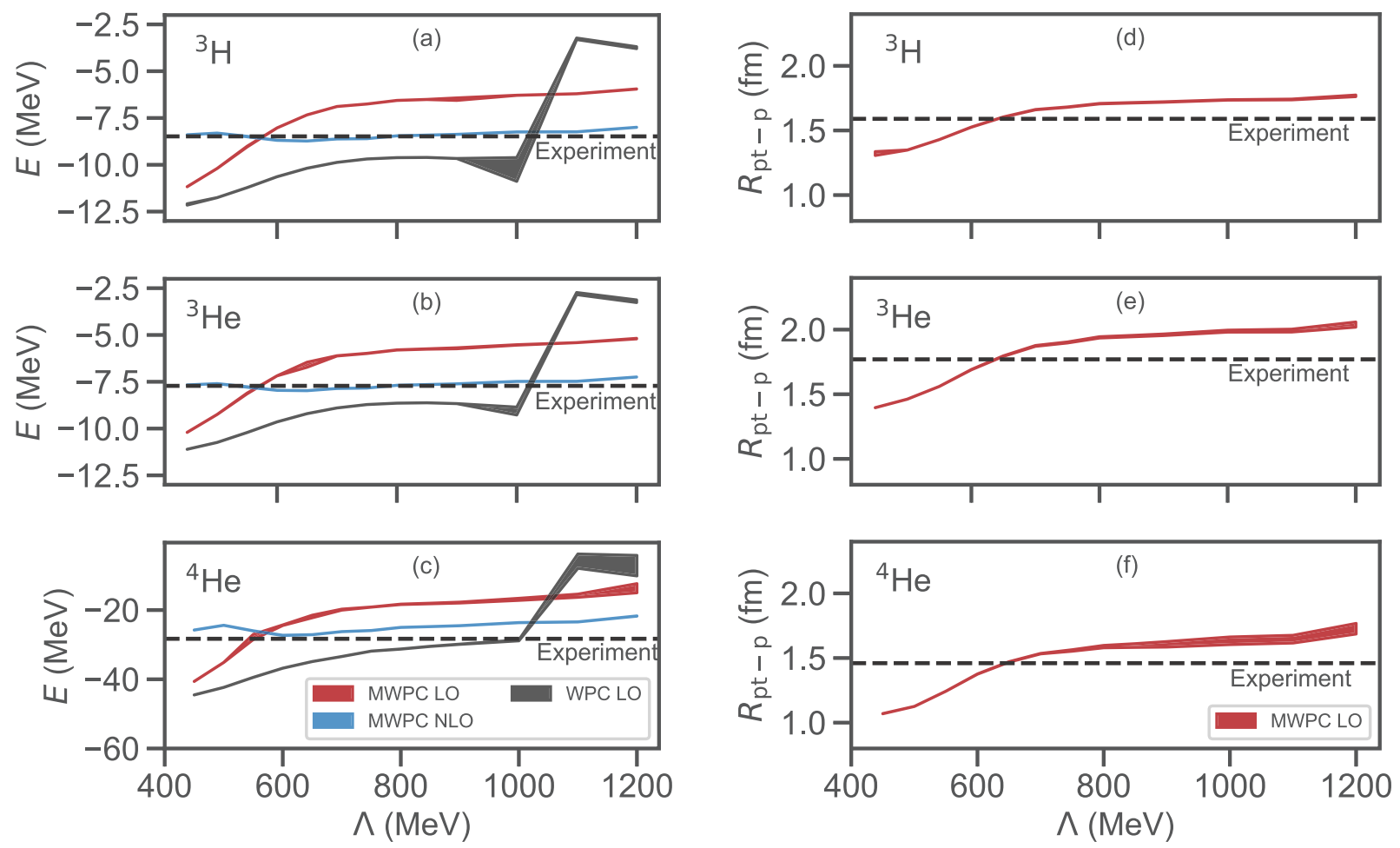

FIG. 6. Ground-state energies [panels (a)-(c)] and point-proton radii [panels (d)-(f)] for ${ }^{3} \mathrm{H},{ }^{3} \mathrm{He}$, and ${ }^{4} \mathrm{He}$ at different values of the regulator-cutoff $\Lambda$. All results are obtained using IR extrapolations of NCSM results for oscillator frequencies $\hbar \Omega \in[35,75] \mathrm{MeV}$ in $61(25)$ major oscillator shells for $A=3(4)$ systems, respectively. The shaded bands indicate the order of magnitude in the uncertainties due to subleading IR corrections. Note the plateaus-indicating RG invariance-with MPWC and the apparent lack thereof for energy results [panels (a)-(c)] based on the WPC interactions, as manifested by the sharp jump around $\Lambda \approx 1000 \mathrm{MeV}$. See the text for details.

Ground-state energies at $\mathrm{LO}$ and NLO, and radii at LO, are obtained using the translationally invariant Jacobi-NCSM method [122] in a harmonic-oscillator basis. For all calculations we employ rather large oscillator frequencies $\hbar \Omega$ such that we can capture the high-energy components of the potential for large values of the ultraviolet regulator cutoff $\Lambda$. To estimate the values of the model-space converged results we extrapolate in the infrared (IR) momentum scale [67-71] using the formalism outlined in Ref. [123]. All extrapolations are based on a set of NCSM calculations carried out for $\hbar \Omega \in[35,75] \mathrm{MeV}$ using 61(25) major oscillator shells for $A=3(4)$, respectively. The extrapolation approach [123] allows an order of magnitude estimate of the magnitude of subleading IR corrections, which we will indicate with an uncertainty band.

The MWPC potentials are known to generate RG-invariant $\mathrm{NN}$ amplitudes. Thus, we expect the numerical values for each observable in our NCSM calculations to exhibit a plateau with respect to large values of the regulator cutoff $\Lambda$. Should this plateau not manifest itself, it would be a clear signature of missing counterterms necessary to absorb the exposed short-range physics at the present order. Such a deficit is clearly visible in, e.g., LO predictions of the ground-state energies in $A=3,4$ systems when using WPC, as shown in the left panels of Fig. 6 . For these calculations, the energies exhibit a clearly noticeable jump at $\Lambda \approx 1000 \mathrm{MeV}$ indicating a possible divergence. This behavior is due to the well-known inconsistency in WPC that originates in the lack of necessary $P$-wave counterterms [41]. Such artifacts are remedied in MWPC, and we find that the binding energies of ${ }^{3} \mathrm{H}$ and ${ }^{3} \mathrm{He}$ indeed exhibit convincing signs of plateaus as $\Lambda \gtrsim 800 \mathrm{MeV}$ at LO and NLO, see the left panels in Fig. 6 . This is in accordance with the known results of the Faddeev calculations presented in Refs. [41,54], where the cutoff could also be taken much larger. We have verified that our LO (NLO) results agree with Song et al. [54,124] when the same $\mathrm{NN}$ input and cutoff are used. It is challenging to converge Jacobi-NCSM calculations for $A=3$ nuclei when using $\Lambda \gtrsim$ $1.2 \mathrm{GeV}$. Using large oscillator frequencies we observe increasing extrapolation uncertainties due to subleading IR corrections.

For ${ }^{4} \mathrm{He}$, the model-space convergence of the NCSM calculations, using interactions with larger cutoffs, are associated with larger uncertainties in the IR extrapolation, see bottom row of panels in Fig. 6. Still, we see the first signs of a RG-invariant description of ${ }^{4} \mathrm{He}$ in MWPC.

We note that the error bands presented in this work do not include any estimate of the order-by-order EFT truncation error. Here, we focus on the prerequisites, i.e., RG invariance, for enabling an EFT-based analysis of the epistemic uncertainty. Nevertheless, the cutoff variation of the results presented in this work serve as a rough handle on the truncation error. More detailed discussions regarding this subject can be found in Refs. [119,120,125-131].

For point-proton radii, we make predictions at $\mathrm{LO}$, as shown in the right panels of Fig. 6. Again, IR extrapola- 
tions were employed following Ref. [123]. The bands indicate rather larger uncertainties from subleading IR corrections, which is consistent with the need to employ large oscillator frequencies in the NCSM. Nevertheless, we observe a similar plateau for radii as for the energies and observe signs of RG-invariant predictions in this observable.

The MWPC results agree rather well with the experimental values, and the size of subleading corrections to the groundstate energy (as seen when going from LO to NLO) is very promising. In fact, up to and including NLO, where additional $S$-wave physics is included, the energies in ${ }^{3} \mathrm{H}$ and ${ }^{3} \mathrm{He}$ reproduce experiment nearly exactly, which also indicates that higher-order contributions should be rather small. The impact of such corrections remains to be explicitly tested. For ${ }^{4} \mathrm{He}$, the higher-order contributions must be slightly larger, which is also expected already in dimensional counting [132]. Overall, MWPC appears to make realistic energy and radius predictions for few-nucleon systems with mass numbers $A \leqslant 4$.

\section{Perturbative calculations in the no-core shell model}

All LO calculations were carried out in a fully nonperturbative fashion, while the NLO results in MWPC were obtained perturbatively. In practice, using the Jacobi-coordinate NCSM code we obtained the NLO results presented above using a procedure based on the Hellmann-Feynman theorem. First, we multiply the NLO interaction potential in Eq. (4) with a small coefficient and subsequently solve the three- and four-body Schrödinger equations nonperturbatively. By examining the results as a function of the small coefficient, the perturbative contribution can be reliably extracted. See, e.g., Sec. IV A in Ref. [133] for the detailed procedure.

It is also possible to directly evaluate the expectation value of first-order perturbation theory. In the NCSM this can be done with minimal modifications by terminating the iterative Lanczos diagonalization after a single matrix-vector multiplication using the LO eigenstate as pivot vector. We implemented this approach in the M-scheme code pANTOINE [123] and verified that the different procedures agree for the $A=3,4$ results. The ability to perform this kind of extraction is a crucial step toward the implementation of any perturbative scheme. Starting from second order in perturbation theory, it is more involved to directly evaluate the perturbative corrections. On the other hand, the Hellmann-Feynman procedure can be carried out to extract the perturbative contribution at arbitrary order without much modification of current NCSM codes.

\section{PREDICTIONS FOR ${ }^{6} \mathrm{Li}$ AND ${ }^{16}$ O USING $\chi$ EFT POTENTIALS IN MODIFIED WEINBERG POWER COUNTING}

In this section we present NCSM and CC predictions for the ground-state energies of ${ }^{6} \mathrm{Li}$ and ${ }^{16} \mathrm{O}$ at $\mathrm{LO}$ and NLO using MWPC. For ${ }^{6} \mathrm{Li}$ we also compute the point-proton radius and the ground-state quadrupole moment at LO. Potentials based on RG-invariant formulations of $\chi \mathrm{EFT}$, e.g., MWPC, have never been employed for predicting nuclei in the $p$ shell or beyond. Our main focus here is to study the evolution of the ground-state energy in selected $A>4$ nuclei as we increase the regulator cutoff $\Lambda$. The enlargement of the cutoff leads to an enhanced ultraviolet part of the potential. In the NCSM this ultraviolet physics must be captured by enlarging the model space, which induces an exponential increase in basis size. We find that it becomes very challenging to converge the ground-state energy and wave function for $A>4$ nuclei with $\Lambda \gtrsim 600 \mathrm{MeV}$. The ultraviolet component also causes difficulties in producing a reasonable reference state for the $\mathrm{CC}$ calculations. In this work we obtained reliable results for ${ }^{6} \mathrm{Li}$ and ${ }^{16} \mathrm{O}$ up to $\Lambda \approx 650$ and $600 \mathrm{MeV}$, respectively.

For nuclei with mass number $A>4$, the effect of $P$ waves becomes more relevant. As outlined in Sec. II, we constructed LO potentials, labeled MWPC(40) and MWPC(200), where the LEC in the ${ }^{3} P_{2}$ wave was renormalized to reproduce phase-shift data in two different ways, see Figs. 2 and 3. The NLO potential in MWPC, see Eq. (4), only affects the ${ }^{1} S_{0}$ wave and is identical for MWPC(40) and MWPC(200). For the MWPC(40) interaction, the phase shifts in the ${ }^{3} P_{2}-{ }^{3} F_{2}$ channel are overly attractive. In contrast, the MWPC(200) potential exhibit more repulsive phase shifts. A detailed study of how the LO description of the ${ }^{1} S_{0}$ phase shifts in $\chi$ EFT impacts nuclear ground-state energies is presented in Sec. V.

\section{A. No-core shell-model calculations of ${ }^{6} \mathrm{Li}$ in modified Weinberg power counting}

As demonstrated in Sec. III, the bulk properties of few-nucleon systems with mass number $A \leqslant 4$ can be described reasonably well using MWPC. Furthermore, the results exhibit signatures of RG-invariance which is a minimal requirement of an EFT. In some ways, ${ }^{6} \mathrm{Li}$ constitutes the simplest nucleus beyond ${ }^{4} \mathrm{He}$. It consists of only two more nucleons, and with the additional proton and neutron naively represented as harmonic oscillator $P$-wave singleparticle states. Here, we perform NCSM calculations of ${ }^{6} \mathrm{Li}$ using the M-scheme code pANTOINE [123] with oscillator basis frequencies $\hbar \Omega \in[30,55] \mathrm{MeV}$ in 20 major oscillator shells $\left(N_{\max }=18\right)$. The relatively large frequencies are needed to improve the ultraviolet convergence for higher values of the regulator cutoff. We study regulator cutoffs $\Lambda \in[450,700] \mathrm{MeV}$ and $\Lambda \in[450,650] \mathrm{MeV}$, in $50 \mathrm{MeV}$ increments, for MWPC(40) and MWPC(200) interactions, respectively. Consequently, we again adopted the infrared extrapolation scheme from Ref. [123]. It should also be noted that the NLO corrections were computed perturbatively with pANTOINE for ${ }^{6} \mathrm{Li}$.

The effects of relative $P$ waves on the ground-state energy of ${ }^{6} \mathrm{Li}$ is obvious when comparing the results for MWPC(40) and MWPC(200) in Fig. 7. Full convergence with respect to $\Lambda$ is not reached for ${ }^{6} \mathrm{Li}$ due to the computational limitations. However, our results indicate that the ground-state at LO is less bound than ${ }^{4} \mathrm{He}$ plus ${ }^{2} \mathrm{H}(\alpha+d$ threshold $)$ obtained with the same interaction once $\Lambda \gtrsim 550$. This is a signature that MWPC does not generate a physical description of the ${ }^{6} \mathrm{Li}$ state, which should be bound with respect to the $\alpha+d$ threshold by nearly $2 \mathrm{MeV}$. We note that this unphysical behavior has been observed also with WPC at LO [134]. Furthermore, this unphysical description appears to persist at NLO. How- 

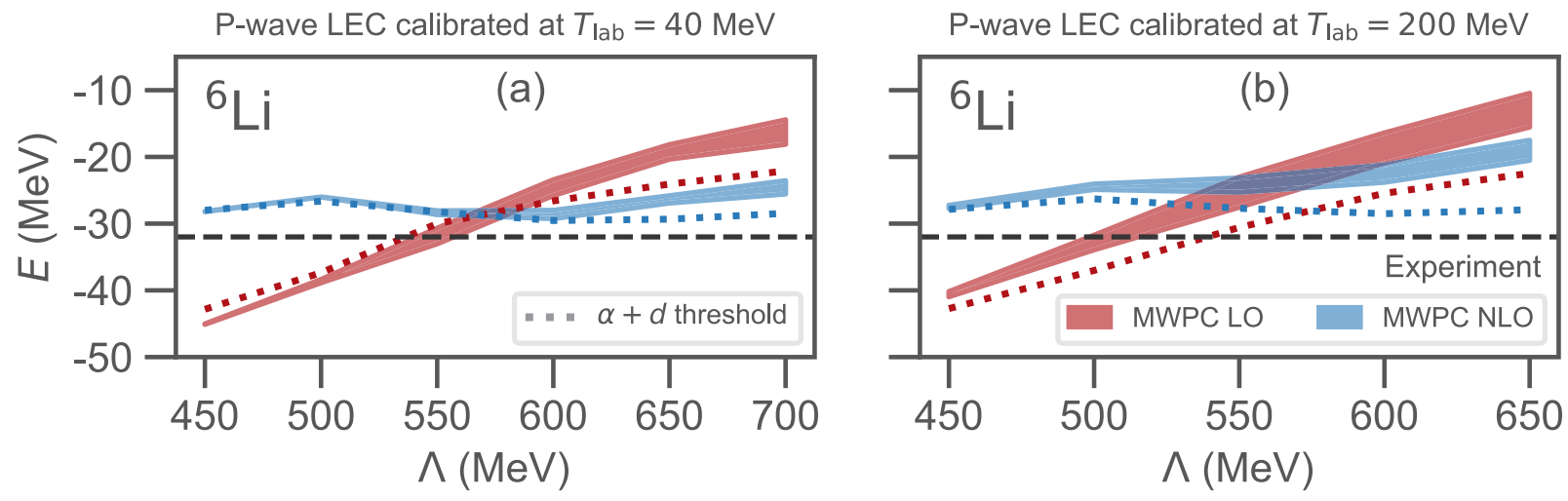

FIG. 7. Ground-state energies $E$ for ${ }^{6} \mathrm{Li}$ at LO and NLO using MWPC(40) [panel (a)] and MWPC(200) [panel (b)] as a function of the regulator cutoff $\Lambda$. The MWPC NLO results exhibit a markedly weaker dependence on the cutoff $\Lambda$ and lie closer to the experimental result (dashed line). The bands indicate the estimated uncertainties from subleading IR corrections. The dotted lines show the $\alpha+d$ threshold (using consistent interactions).

ever, some care is needed when interpreting our results. The NCSM method includes all particle-hole excitations in the model space, and within IR uncertainties, the ground-state energy should at least reside on the threshold. The difference between the envelope of the IR uncertainty band and the threshold indicates that the extrapolation error is underestimated-at least for larger values of the cutoff $\Lambda$. We also note that the ground-state energies obtained with MWPC(40) resides below the $\alpha+d$ threshold for $\Lambda \leqslant 550 \mathrm{MeV}$, and cross the threshold at $\approx 600 \mathrm{MeV}$, where we still consider our NCSM results to be reasonably well-converged. In fact, for both MWPC(40) and MWPC(200), the decreasing rate of ${ }^{6} \mathrm{Li}$ binding against cutoff at LO appears to be linear (with fixed slopes) before and right after crossing the threshold. Thus, one could not infer any obvious shift in the wave functions-which would be a signature of a sudden change in the pole structure. As a result, rather than immediately concluding that something is fundamentally wrong on the PC side, we cannot rule out that the apparent failure of MWPC is simply an effect of fine tuning in the LECs. Nevertheless, the predicted LO ground-state energy for $\Lambda \geqslant 600(E \lesssim-15$ $\mathrm{MeV})$ is far from the experimental value $-32 \mathrm{MeV}$. This strong underbinding implies that the effect of higher orders in MWPC must be sizable, and this points to the possible need for some modification of the LO potential in MWPC.

Given our model-space restrictions, and the consequent use of large oscillator frequencies, it is challenging to reach converged predictions for the point-proton radius of ${ }^{6} \mathrm{Li}$. The estimated uncertainties coming from the IR extrapolation are sizable, see Fig. 8. We note that the radius results for $\Lambda \gtrsim$ $550 \mathrm{MeV}$ are unphysical in the sense that we quote a finite radius for an unbound system. Indeed, for $\Lambda \gtrsim 550 \mathrm{MeV}$ the obtained ground-state energy for ${ }^{6} \mathrm{Li}$ is above the $\alpha+d$ threshold and the NCSM basis truncation imposes an IR cutoff that limits the radius prediction. For $\Lambda \leqslant 550 \mathrm{MeV}$, with MWPC(200) the radius is predicted slightly larger, and also closer to experiment. This behavior is intuitively consistent with the slightly lower binding generated by this interaction. Overall, our results indicate that the predicted radius of ${ }^{6} \mathrm{Li}$ is too small compared with experiment. This corresponds to a too large central density of ${ }^{6} \mathrm{Li}$. If this persists to other nuclei, it implies a too large saturation density of nuclear matter, which is a well-known problem $[88,135]$ in nuclear structure theory that seems to persist when using MWPC. Recent analyses suggest that this problem might be resolved by the explicit inclusion of the $\Delta(1232)$ degree of freedom in $\chi$ EFT $[89,90,136]$.

Finally, we also studied the quadrupole moment of the ${ }^{6} \mathrm{Li}$ ground state, which is experimentally known to be very small and negative [137] $Q=-0.0818(17) e \mathrm{fm}^{2}$. This small value results from a cancellation of wave-function components and is consequently very sensitive to details of the nuclear structure. The small quadrupole moment has been successfully reproduced with ab initio NCSM calculations using phenomenological, realistic NN interactions [138]. Using the MWPC(200) LO interaction from this work we find, however, that we obtain a large positive quadrupole moment for $\Lambda=450 \mathrm{MeV}$, a small one for $\Lambda=500 \mathrm{MeV}$, and a negative one for $\Lambda=550 \mathrm{MeV}$. The evolution of the predicted quadrupole moment as a function of the regulator cutoff points in the direction of varying single-particle structures. This finding will also be verified with the ${ }^{16} \mathrm{O}$ results in the next section. We note that a full convergence study remains to be performed, but that the observed trend is robust with the respect to changes in the oscillator frequency and the size of the model space.

\section{B. ${ }^{16} \mathrm{O}$ in modified Weinberg power counting}

We now turn to the case of the doubly magic nucleus ${ }^{16} \mathrm{O}$ and calculate its ground-state energy using interactions from MWPC at LO and NLO. The oxygen isotopic chain has been extensively studied with $a b$ initio methods and chiral potentials in WPC $[87,88,139-145]$. These calculations have revealed that an accurate description of binding energies, radii, and spectra is very sensitive to fine details of the employed chiral potential model. Furthermore, in Ref. [119] it was found that simultaneously optimized chiral NN and NNN interactions from WPC at NNLO predicts ${ }^{16} \mathrm{O}$ to be unbound with respect to decay into four $\alpha$ particles. Interestingly, recent 

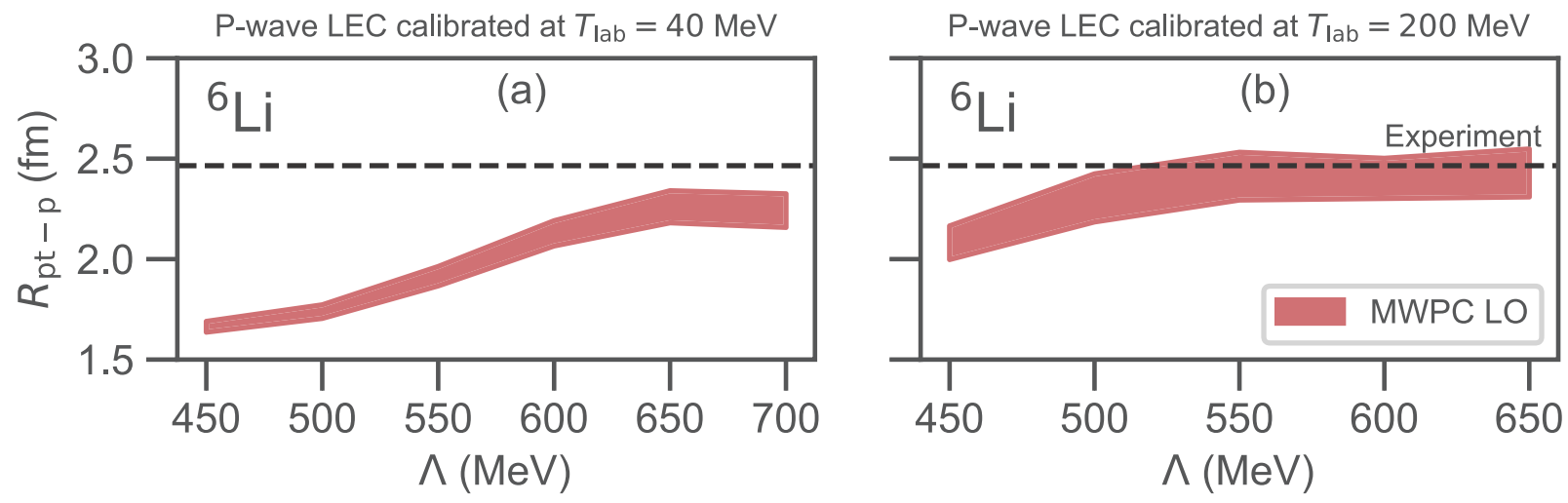

FIG. 8. Point-proton radii $R_{p t-p}$ for ${ }^{6} \mathrm{Li}$ at LO using MWPC(40) [panel (a)] and MWPC(200) [panel (b)] as a function of the regulator cutoff $\Lambda$. The bands indicate the estimated uncertainties from subleading IR corrections. The dashed line marks the point-proton radius corresponding to the experimentally determined charge radius.

calculations $[89,90]$ based on chiral potentials with explicit inclusion of $\Delta$ isobars at NNLO found ${ }^{16} \mathrm{O}$ to be bound. This result might also indicate an important role of the finite nucleon size for reproducing saturation properties in nuclei.

We will use single-reference $a b$ initio CC theory to calculate the ground state of ${ }^{16} \mathrm{O}$. The many-nucleon wave function is represented via an exponential ansatz $|\Psi\rangle=e^{T}\left|\Phi_{0}\right\rangle$, where $\left|\Phi_{0}\right\rangle$ is an uncorrelated reference state commonly chosen as the Hartree-Fock (HF) ground-state. Many-body correlations are then included by acting with $e^{T}$ on the reference state, where $T=T_{1}+T_{2}+\cdots$ is a linear expansion in particlehole excitations typically truncated at some low excitation rank. In this work we truncate $T$ at the singles-doubles excitation level. When using spherical CC, we also include triples excitations perturbatively in an approach known as the $\Lambda$-CCSD(T) approximation [21,146,147]. For closed-shell systems that can be well described using a single-reference formalism, this approximation has been shown to account for about $99 \%$ of the full correlation energy [62]. We remind the reader that the $\mathrm{CC}$ method is nonvariational, and as a consequence the Hellmann-Feynman theorem is strictly not valid when evaluating expectation values when the cluster operator $T$ is truncated (see, e.g., Ref. [147] for more details). We therefore compute the perturbative corrections at NLO as an expectation value using the $\mathrm{LO} \mathrm{CC}$ wave function.

With MWPC(40) at LO we find that the Hartree-Fock (HF) single-particle orbitals exhibit an unconventional ordering with a $1 d_{5 / 2}$ orbital below the $1 p_{1 / 2}$ orbital and a very large $(\geqslant 80 \mathrm{MeV})$ splitting between the $1 p_{1 / 2}$ and $1 p_{3 / 2}$ orbitals. Although these single-particle orbitals are not observable quantities [148], the observed ordering is in stark contrast with the traditional single-particle shell-model picture of Mayer [149], which usually provides a realistic starting point for describing well-bound nuclei near the valley of beta stability. This untraditional ordering is presumably caused by the over-attractive ${ }^{1} S_{0}$ and ${ }^{3} P_{2}$ partial waves, as shown in Figs. 1 and 2. Furthermore, the inversion of the $d_{5 / 2}$ and $p_{1 / 2}$ orbitals prevents us from a spherical single-reference $\mathrm{CC}$ description of the ground state of ${ }^{16} \mathrm{O}$. To compute the ground state of ${ }^{16} \mathrm{O}$ using MWPC(40) we therefore performed CC calculations starting from an axially deformed Hartree-Fock reference state. Here the Hartree-Fock reference state was constructed by assuming prolate deformation, see Ref. [150] for more details. The ground-state energies are plotted as a function of the cutoff $\Lambda$ in Fig. 9. Being a doubly magic nucleus, ${ }^{16} \mathrm{O}$ should be spherical in its ground state. Thus we conclude that the MWPC(40) LO interaction is highly unphysical. Also, at lower cutoffs, the LO result yields a ground-state energy that is two orders of magnitude from the experimental value. The unphysical LO results do not motivate a further study of the NLO corrections.

We performed the calculations using model-space sizes of up to 11 major oscillator shells $\left(N_{\max }=10\right)$ and varied the oscillator frequencies over a wide range $(\hbar \Omega \in[35,60] \mathrm{MeV})$. This allowed us to find the energy minimum for a given model space and extract reasonably-well-converged groundstate energies. For example, for the hardest interaction, $\Lambda=$ $600 \mathrm{MeV}$, the CCSD energy at the $\hbar \Omega$ minimum goes from

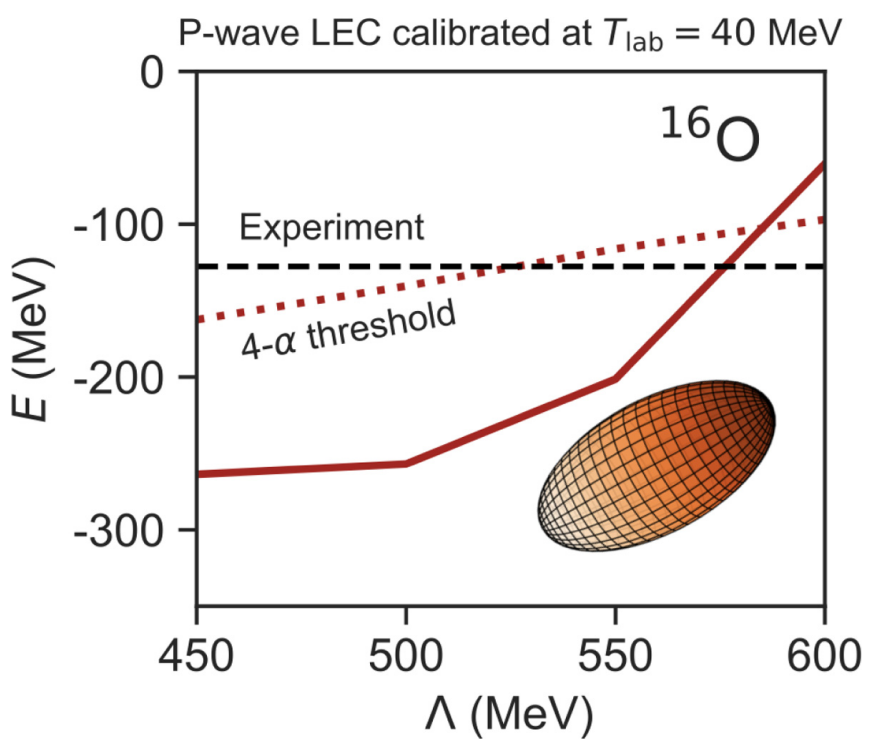

FIG. 9. The ground-state energy of ${ }^{16} \mathrm{O}$ versus regulator cutoff $\Lambda$ at LO in MWPC(40). The ground state is axially deformed for all values of the cutoff in this figure. 
$-58 \mathrm{MeV}$ to $-61 \mathrm{MeV}$ when increasing from $N_{\max }=8$ to $N_{\text {max }}=10$. We note that at $\Lambda=600 \mathrm{MeV},{ }^{16} \mathrm{O}$ becomes unstable against decay into four $\alpha$ particles. A CC prediction for the ground-state of ${ }^{16} \mathrm{O}$ that is unbound with respect to the four- $\alpha$ decay threshold requires some care in its interpretation. Clearly, such an energy does not represent the true ground state of the system. Indeed, four $\alpha$ particles very far apart would yield an energy equal to the corresponding threshold value. But this very exotic $\alpha$-cluster configuration cannot be described in the $\mathrm{CC}$ approach that we use. To describe a state that is dominated by clusterization into $\alpha$ particles, one would need to include at least $4 \mathrm{p}-4 \mathrm{~h}$ excitations in the cluster amplitude $T$. Such an approach is currently not possible due to the orders of magnitude increase in computational cost.

We now move on to the MWPC(200) interaction, which has a more repulsive ${ }^{3} P_{2}$ component with a better overall agreement with the Nijmegen phase shifts analysis. We note that there is still an over-attraction in the $\mathrm{NN}^{1} S_{0}$ channel as shown in Fig. 1. With MWPC(200), we find a conventional ordering of single-particle states. All CC calculations indicate that the spherical states of ${ }^{16} \mathrm{O}$ are always more bound than their corresponding deformed counterparts throughout $\Lambda=$ 450-600 MeV for this interaction. However, some pathological behavior is still present. Most importantly, we find that, for cutoff values $\Lambda>450$, the ground state in ${ }^{16} \mathrm{O}$ is always unstable with respect to decay into four $\alpha$ particles. Our spherical CC calculations were carried out in a model space up to 17 major oscillator shells $\left(N_{\max }=16\right)$. The results are very similar to those plotted later in the left panel of Fig. 12 (i.e., MWPC(200) with perturbative $P$ waves).

There are most likely several possible origins that contribute to the failures of MWPC(40) and MWPC(200) in producing a physical ${ }^{16} \mathrm{O}$ ground state. First, as already seen in the case of ${ }^{6} \mathrm{Li}$, the effects due to different strategies for calibrating the LO LEC in the ${ }^{3} P_{2}-{ }^{3} F_{2}$ partial waves are further magnified in ${ }^{16} \mathrm{O}$. For example, at $\Lambda=450 \mathrm{MeV}$, MWPC(40) and MWPC(200) yield vastly different shapes and energies for the ground state; $-264 \mathrm{MeV}$ (deformed) and $-150 \mathrm{MeV}$ (spherical), respectively. From the results presented above, we have to conclude that MWPC cannot be employed for realistic predictions of atomic nuclei beyond ${ }^{4} \mathrm{He}$. However, we would like to point out that it is possible to obtain a remarkably good descriptions of the ground-state energies of ${ }^{4} \mathrm{He}$ as well as ${ }^{16} \mathrm{O}$ at LO in MWPC if one tunes the regulator cutoff $\Lambda=280$ $\mathrm{MeV}$. At this value, the LO description of the ${ }^{1} S_{0}$ phase shift is qualitatively very similar to Nijmegen data and the NLO correction is small, see Fig. 10. This particular LO interaction yields ${ }^{16} \mathrm{O}$ and ${ }^{4} \mathrm{He}$ binding energies 127.3 and $29.5 \mathrm{MeV}$, respectively. The ${ }^{16} \mathrm{O}$ ground state is also spherical. Using the regulator cutoff $\Lambda$ as one of the fitting parameters in this way eliminates the model-independent aspects of $\chi$ EFT and its fundamental connection with QCD will be lost. Of course, an interaction with a tuned regulator cutoff could still be useful for guiding experiment and phenomenology. However, in a $\chi$ EFT with a PC that yields RG-invariant observables, no particular cutoff is preferred. One can choose any value $\Lambda \gtrsim \Lambda_{b}$ for a quantitative calculation, and then try to estimate residual, i.e., higher-order, cutoff dependencies and EFT errors $[125,126]$. Setting $\Lambda=280 \mathrm{MeV}$, which is most likely

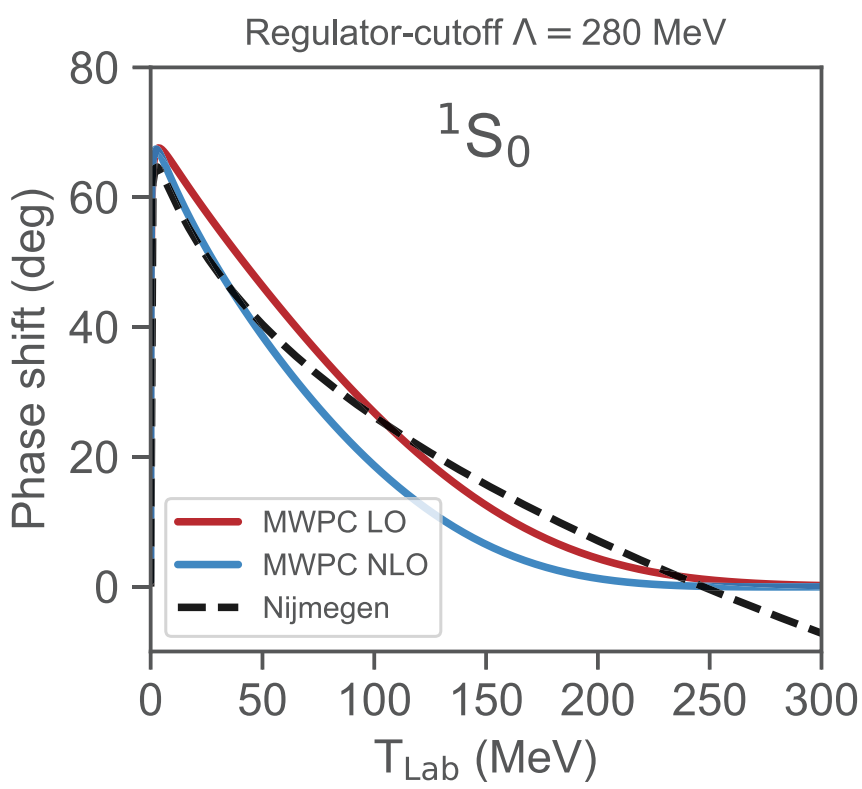

FIG. 10. MWPC LO and NLO ${ }^{1} S_{0}$ phase shifts using a regulator cutoff $\Lambda=280 \mathrm{MeV}$ as a function of laboratory scattering energy $T_{\mathrm{Lab}}$. The MWPC LO interaction yields slightly more attractive phase shifts.

much smaller than the breakdown scale $\Lambda_{b}$ of $\chi$ EFT, removes a large chunk of the relevant low-momentum dynamics from the loops. As such, this model will probably extrapolate unreliably to larger mass numbers $A$ and it will be difficult to assign a physics-based EFT uncertainty to the results.

\section{PREDICTIONS BASED ON OTHER RENORMALIZATION-GROUP-INVARIANT POWER-COUNTING SCHEMES}

The results for ${ }^{6} \mathrm{Li}$ and ${ }^{16} \mathrm{O}$ presented in the previous sections most likely rule out the usefulness of MWPC for heavier systems. Obviously, one needs to seek alternative PCs. In fact, the large discrepancy between the Nijmegen analysis and the LO ${ }^{1} S_{0}$ phase shift as shown in Fig. 1 has already motivated research on several such alternative PCs.

\section{A. The dibaryon field}

Potentials in $\chi$ EFT that employ the dibaryon (db) auxiliary field $[45,51,151]$ give a very good and RG-invariant description of the ${ }^{1} S_{0}$ phase shift at LO. However, in such approaches, the resulting potential contains an energy-dependent shortrange term

$$
V_{\mathrm{db}}^{\mathrm{LO}}(E)=\frac{1}{\Delta+c E}
$$

where the on-shell energy $E=k_{0}^{2} / m_{N}$, with $k_{0}$ denoting the on-shell center-of-mass momentum, and $m_{N}=938.9 \mathrm{MeV}$ denoting the nucleon mass. The two parameters $\Delta$ and $c$ are LECs to be renormalized. Note that, in order to reproduce the amplitude zero at $T_{\mathrm{Lab}} \approx 250 \mathrm{MeV}$, the $\mathrm{PC}$ proposed in Ref. [45] incorporates one more LEC which effectively has 
the same structure as the usual $C_{1_{S_{0}}}$ contact term, i.e.,

$$
V_{\mathrm{DBZ}}(E)=C_{\mathrm{i}_{S_{0}}}+V_{\mathrm{db}}^{\mathrm{LO}}(E) \text {. }
$$

We denote this PC as DBZ (dibaryon potential which reproduces the amplitude zero). The long-range part of the OPE potential is then iterated nonperturbatively together with the short-range $V_{\mathrm{DBZ}}$ potential in the Lippmann-Schwinger or Schrödinger equations.

In the NN sector, solutions of the Lippmann-Schwinger or Schrödinger equations based on an energy-dependent potential can be obtained straightforwardly. The only caveat is that eigenfunctions are no longer necessarily orthogonal $[152,153]$. On the other hand, it is very difficult to solve a many-nucleon Schrödinger equation based on energydependent potentials. To proceed, approximations are needed. We studied the predictions from energy-dependent DBZ potentials for describing $A=3,4$ systems, and tried to quantify the uncertainties due to nonunique transformations of an energy-dependent potential to a purely momentum-dependent potential $V\left(p, p^{\prime}\right)$. We adopted the following procedure. First, we solve the two-body Schrödinger equation

$$
\left[H_{0}+V(E)\right]|\psi\rangle=E|\psi\rangle,
$$

with $H_{0}$ being the kinetic energy, $\psi$ the eigenfunction, and $V(E)$ the LO potential which contains both momentum and energy dependencies. We represent the total Hamiltonian $H$ in a finite momentum basis with $N \approx 100$ states that cover momenta $\left[0, \Lambda_{p}\right]$ and where we also ensure that $\Lambda_{p}>\Lambda$. Thereafter, it is straightforward to solve Eq. (7) for $N$ eigenvectors iteratively until the corresponding difference between the on-shell energy $E$ on both sides of the equation falls below a convergence criterion $\varepsilon_{E}=10^{-16}$. The resulting selfconsistent eigenfunctions and eigenvalues are denoted as $\psi_{E_{i}}$ and $E_{i}$. Due to the energy dependence, the span of eigenvectors $\left\{\psi_{E_{i}}\right\}_{i=1}^{N}$ does not form an orthogonal basis. Instead, we choose to employ the Gram-Schmidt orthogonalization method to form an orthogonal basis $\left\{\psi_{E_{i}}^{\mathrm{GS}}\right\}_{i=1}^{N}$. This transformation is not unique. Indeed, we can start from any of the $N$ vectors in the Gram-Schmidt procedure and generate a different basis. However, equipped with any orthogonal basis we can reconstruct an on-shell equivalent Hamiltonian

$$
\begin{aligned}
\left\langle p|H| p^{\prime}\right\rangle & =\sum_{E_{i}} \mathcal{Y}_{E_{i}^{\prime}}\left\langle p \mid \psi_{E_{i}}^{\mathrm{GS}}\right\rangle\left\langle\psi_{E_{i}}^{\mathrm{GS}}|H| \psi_{E_{i}^{\prime}}^{\mathrm{GS}}\right\rangle\left\langle\psi_{E_{i}^{\prime}}^{\mathrm{GS}} \mid p^{\prime}\right\rangle \\
& =\underbrace{}_{E_{i}}\left\langle p \mid \psi_{E_{i}}^{\mathrm{GS}}\right\rangle E_{i}\left\langle\psi_{E_{i}}^{\mathrm{GS}} \mid p^{\prime}\right\rangle .
\end{aligned}
$$

Subtracting the kinetic term yields a momentum-dependent potential which preserves all of the original eigenvalues

$$
V\left(p, p^{\prime}\right)=\left\langle p|H| p^{\prime}\right\rangle-\frac{p^{2}}{m_{N}} \delta_{p p^{\prime}}
$$

and which we then use in the many-body calculations. After renormalization, the numerical value of $c$ in Eq. (5) turns out to be of order $10^{-4}$ smaller than $\Delta$ and $C_{\mathrm{I}_{0}},{ }^{3}$, which likely

\footnotetext{
${ }^{3}$ Here the three LECs are fit to reproduce $a_{0}=-23.7 \mathrm{fm}, r_{0}=$ $2.7 \mathrm{fm}$, and the Nijmegen phase shift analysis at $T_{\mathrm{Lab}}=250 \mathrm{MeV}$.
}

correspond to a small nonorthogonality between the vectors $\psi_{E_{i}}$. On the other hand, we found that, without any GramSchmidt re-orthogonalization, the resulting $V\left(p, p^{\prime}\right)$ potential will generate phase shifts which deviate about $15 \%$ from the original values, i.e., those given by $V(E)$. The nonuniqueness of the re-orthogonalization procedure is manifested in the momentum-dependent potential as off-shell modifications. The size of this effect can be explored by selecting different $\psi_{E_{i}}$ as the initial vector in the Gram-Schmidt procedure to generate potentials $V\left(p, p^{\prime}\right)$ with differing off-shell behavior. We have carried out this test and find that this effect is propagated into many-body calculations and gives about $10 \%(20 \%)$ variation in the ground-state energies of ${ }^{3} \mathrm{H}\left({ }^{4} \mathrm{He}\right)$. Besides this variation due to the energy dependence of the potential, we find that the reproduction of the corresponding ground-state energies is comparable to the MWPC result, i.e., a slight underbinding. The main difference is that the NLO correction to the energy appears to be smaller, which can be expected judging from the NLO correction at the NN phase-shift level [45]. We also note that shuffling the order of eigenvectors in the Gram-Schmidt procedure creates discontinuities in the first derivative of $V\left(p, p^{\prime}\right)$ with respect to $p$ and $p^{\prime}$. Although this is not forbidden in principle, it could create artifacts of numerical origin in many-body calculations.

The evaluation of DBZ at NLO is even more involved compared with LO because the NLO interactions again contain additional energy dependencies, which read

$$
C_{2}^{\mathrm{DBZ}}+D_{2}^{\mathrm{DBZ}} E+\alpha V_{\mathrm{DBZ}}^{\mathrm{LO}}(E)+\beta\left[V_{\mathrm{DBZ}}^{\mathrm{LO}}(E)\right]^{2},
$$

with $C_{2}^{\mathrm{DBZ}}, D_{2}^{\mathrm{DBZ}}, \alpha$, and $\beta$ denoting four new LECs, and $V_{\mathrm{DBZ}}^{\mathrm{LO}}$ being the short-range LO potential as defined in Eq. (6) (which is not refitted at NLO). A direct perturbative evaluation in the NN sector is straightforward. However, the renormalized interaction is energy dependent and cannot easily be used in many-body calculations. To our knowledge, there is no strict phase-shift equivalent transformation method to be applied perturbatively, as in the NN case. There are several approximate ways to transform the energy-dependent NLO terms in the DBZ potential to a purely momentum-dependent representation. The bottom line in all such methods is to treat the energy-dependent terms in Eq. (11) as small perturbations to the LO amplitude. Once all the energy-dependent terms are transformed (either one by one individually or as a whole) into purely momentum-dependent terms, one must renormalize the LECs associated with those transformed (i.e., momentum-dependent) contact terms at NLO. One could also test the possibility of approximating $E$ by $\frac{p^{2}+p^{\prime 2}}{m_{N}}$ for the second term of Eq. (11), inspired by the equation of motion. In all our attempts to transform the energy dependence to a pure momentum dependence, the four NLO LECs always yield phase-shift-equivalent results, as expected. The off-shell differences, however, manifest themselves in an uncontrollable fashion in many-body calculations. Our analysis indicates that the approximate and nonunique transformation of the energy-dependent DBZ potential at LO yield sizable errors that increase with mass number $A$. We do not present any detailed $\mathrm{CC}$ results for ${ }^{16} \mathrm{O}$. We only summarize that, in all our calculations based on the DBZ potential at LO and NLO, 


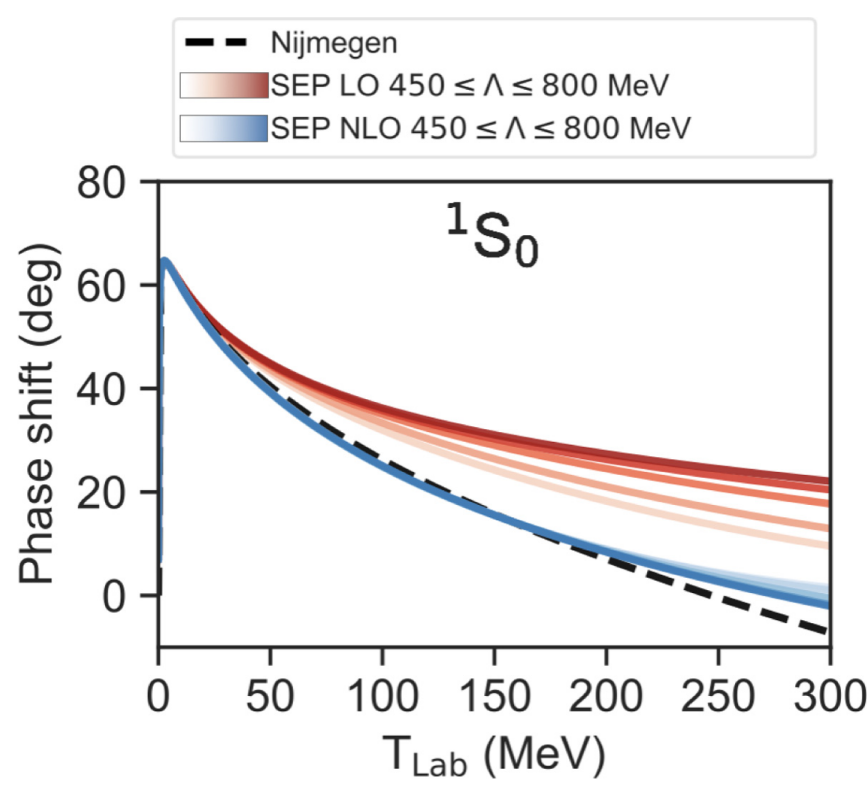

FIG. 11. LO and NLO ${ }^{1} S_{0}$ phase shifts obtained using the power counting based on a separable potential (SEP) defined in Ref. [79] as a function of laboratory scattering energy $T_{\mathrm{Lab}}$. Darker line colors correspond to larger values of the cutoff $\Lambda$. The SEP LO interaction exhibits a stronger cutoff variation in the ${ }^{1} S_{0}$ phase shift compared with the SEP NLO interaction. The latter interaction best reproduces the Nijmegen result (dashed line). The LO LECs are renormalized to the scattering length $a_{0}=-23.7 \mathrm{fm}$ and the effective range $r_{0}=2.7$ $\mathrm{fm}$. The NLO LECs are renormalized to the scattering length $a_{0}=$ $-23.7 \mathrm{fm}$, the effective range $r_{0}=2.7 \mathrm{fm}$ and a best fit to Nijmegen phase shift up to $T_{\mathrm{Lab}}=200 \mathrm{MeV}$.

we never recovered a ${ }^{16} \mathrm{O}$ nucleus that was bound with respect to four- $\alpha$ decay for any values of the regulator cutoff $\Lambda \geqslant 500 \mathrm{MeV}$.

\section{B. A separable potential}

In an effort to eliminate the energy dependence of the dibaryon field while trying to maintain the good reproduction of the ${ }^{1} S_{0}$ phase shift, one could transform the dibaryon structure in the Lagrangian to yield an energy-independent and separable potential (SEP) [154]. The LO short-range structure of this potential reads [79]

$$
V_{\mathrm{SEP}}\left(p, p^{\prime}\right)=\frac{y m_{N}}{\sqrt{p^{2}+m_{N} \Delta} \sqrt{p^{\prime 2}+m_{N} \Delta}} .
$$

This reproduces the short-range physics of one dibaryon field. There are two LECs, $y$ and $\Delta$, at LO. The full LO SEP potential contains the above short-range part plus the Yukawa potential. The resulting ${ }^{1} S_{0}$ phase shifts at LO and NLO are given in Fig. 11.

To generate the NLO amplitude, one perturbatively inserts the following NLO short-range terms in the ${ }^{1} S_{0}$ channel

$$
C+y^{(1)} V_{\mathrm{SEP}}+\Delta^{(1)}\left(\frac{1}{p^{2}+m_{N} \Delta}+\frac{1}{p^{\prime 2}+m_{N} \Delta}\right) V_{\mathrm{SEP}}
$$

where $C, y^{(1)}$, and $\Delta^{(1)}$ are additional LECs, renormalized to provide $a_{0}=-23.7 \mathrm{fm}, r_{0}=2.7 \mathrm{fm}$, and a best fit to the
Nijmegen phase shift up to $T_{\mathrm{Lab}}=200 \mathrm{MeV}$. Note that $V_{\mathrm{SEP}}$ here is the short-range potential already renormalized at LO. The LECs within $V_{\text {SEP }}$ are not refitted at NLO. The resulting NLO phase shifts reproduce the Nijmegen ${ }^{1} S_{0}$ phase shifts quite well for a wide range of cutoff values $\Lambda$ as listed in Fig. 11, which also shows a more reasonable LO to NLO change comparing with MWPC.

As for MWPC and DBZ, the SEP potential yields a reasonable LO prediction of the ground-state energies of ${ }^{3} \mathrm{H},{ }^{3} \mathrm{He}$, and ${ }^{4} \mathrm{He}$, with NLO corrections of expected sizes. In fact, this seems to be a trend; most RG-invariant PCs yield LO and NLO potentials in $\chi$ EFT capable of describing $A \leqslant 4$ nuclei rather well. As for DBZ, the small NLO correction to the ground-state energy using the SEP PC can be inferred from the fact that the space for improvement is tiny since the LO results reproduce NN phase shifts in low partial waves quite well. Note that our results for $A=3,4$ systems at lower cutoffs are in agreement with a recent calculation [155], where the LO treatment in the ${ }^{1} S_{0}$ channel is equivalent to our SEP potential plus one constant contact term.

Having removed the energy dependence, via the separable formulation, we used the SEP potential in $a b$ initio CC calculations to predict the ground-state energy of ${ }^{16} \mathrm{O}$ at LO and NLO for cutoff values $\Lambda \leqslant 600 \mathrm{MeV}$. We refer to the potentials associated with the LEC in ${ }^{3} P_{2}-{ }^{3} F_{2}$ channels fit up to $T_{\mathrm{Lab}}=40$ and $T_{\mathrm{Lab}}=200 \mathrm{MeV}$ as $\operatorname{SEP}(40)$ and $\operatorname{SEP}(200)$, respectively.

For SEP(40), we found that the over-attractive ${ }^{3} P_{2}$ partial wave still generates an ordering of the HF single-particle states that is in stark contrast to traditional shell-model interpretations, or gives a very large splitting between the $1 p_{1 / 2}$ and $1 p_{3 / 2}$ states. On these grounds, we discard further analyses of the SEP(40) interaction. For SEP(200), the singleparticle states exhibit a conventional ordering, which suggests a spherical ground state. However, we are not able to obtain ground-state energies of ${ }^{16} \mathrm{O}$ below the corresponding four- $\alpha$ threshold throughout the cutoff range $\Lambda=450-600 \mathrm{MeV}$. Although this renders the NLO correction less meaningful, we note that they are always repulsive-which makes the results even more unphysical.

\section{Perturbative $P$ waves}

It was shown recently [53] that all of the $P$-wave amplitudes, with the exception of ${ }^{3} P_{0}$, can be reproduced rather well in an order-by-order perturbative approach. This particular $P$ wave still requires a nonperturbative treatment. ${ }^{4}$ Thus, it is possible that the LO amplitude in $\chi$ EFT should only comprise the ${ }^{1} S_{0},{ }^{3} S_{1}-{ }^{3} D_{1}$, and ${ }^{3} P_{0}$ waves. To explore the consequences of this recent $\mathrm{PC}$, we performed many-body calculations for the ground-state energies in ${ }^{3} \mathrm{H},{ }^{3} \mathrm{He}$, and ${ }^{4} \mathrm{He}$ using the Jacobi-NCSM method and ${ }^{16} \mathrm{O}$ using the $\mathrm{CC}$ method based on MWPC and SEP potentials with perturbative $P$ waves. The LEC in the ${ }^{3} P_{0}$ channel is renormalized to Nijmegen phase

${ }^{4}$ For the ${ }^{3} P_{0}$ channel, a further study [156] suggests that a perturbative treatment is possible if an additional counterterm is promoted to $\mathrm{LO}$ in addition to the long-range OPE in this channel. 

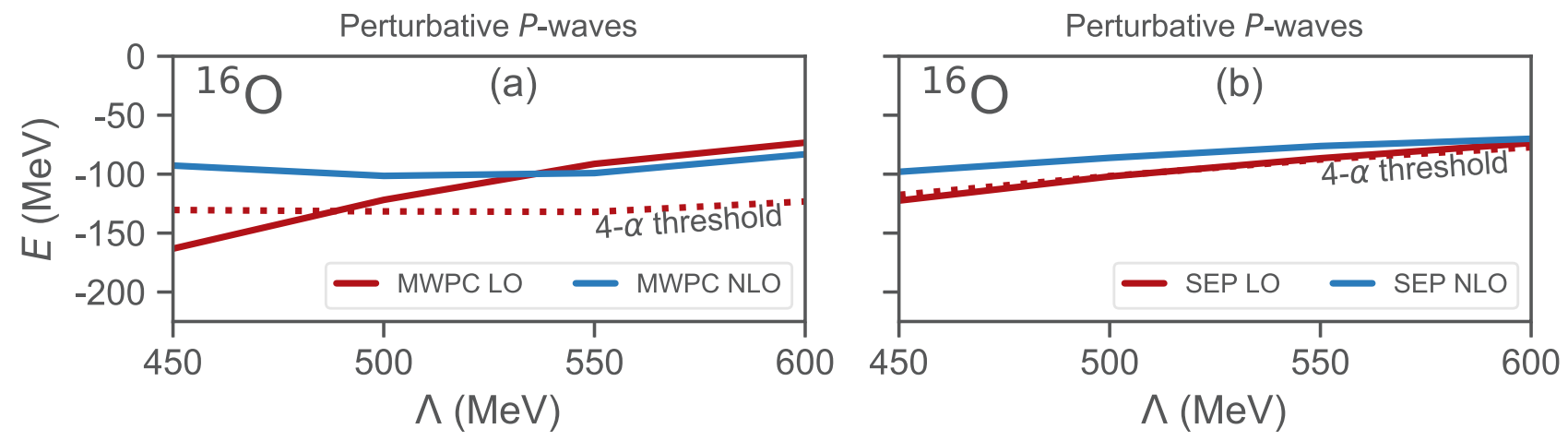

FIG. 12. Ground-state energy of ${ }^{16} \mathrm{O}$ versus regulator cutoff $\Lambda$ with perturbative $P$ waves at LO and NLO using MWPC [panel (a)] and SEP PC [panel (b)]. The respective LO four- $\alpha$ thresholds are indicated by the red dotted lines. The NLO results in both panels exhibit a weaker dependence with respect to variations in the cutoff $\Lambda$.

shifts up to $T_{\mathrm{Lab}}=40 \mathrm{MeV}$ as before, and the interaction in all remaining $P$ waves (including channels coupled to ${ }^{3} P_{2}$ ) vanish identically at LO. Since there can no longer be any differences in the ${ }^{3} P_{2}-{ }^{3} F_{2}$ channels due to alternative calibration procedures, we can drop the (40) and (200) labels from such interactions.

With perturbative $P$ waves, the ${ }^{3} \mathrm{H}$ and ${ }^{3,4} \mathrm{He}$ binding energies reside between the MWPC(40) and MWPC(200), or SEP(40) and SEP(200) if the SEP PC is adopted in the ${ }^{1} S_{0}$ channel. We also observe similar convergence patterns as before when going from LO to NLO. This is expected since nuclei with mass number $A \leqslant 4$ are quite insensitive to $P$ waves. Indeed, all our previous results based on the two different ${ }^{3} P_{2}-{ }^{3} F_{2}$ calibrations differed at most $5 \%$ for the cutoff values considered in this work.

Turning to ${ }^{16} \mathrm{O}$, we found that the Hartree-Fock solutions starting from MWPC and SEP PC with perturbative $P$ waves give single-particle states with ordering that allows for a spherical single-reference $\mathrm{CC}$ description. We therefore computed the ground-state energies of ${ }^{16} \mathrm{O}$ using the spherical $\Lambda \mathrm{CCSD}(\mathrm{T})$ approximation, and the results are presented in Fig. 12. We employed 17 major oscillator shells $\left(N_{\max }=16\right)$ and extract a minimum CC energy for $\hbar \Omega \in[16,50] \mathrm{MeV}$ for all values of the cutoff $\Lambda=450-600 \mathrm{MeV}$. As one can see, MWPC with perturbative $P$ waves yields a ${ }^{16} \mathrm{O}$ ground state that decays into four $\alpha$ particles for $\Lambda>500 \mathrm{MeV}$. For SEP with perturbative $P$ waves, the CC results for the ground-state energy borders the four- $\alpha$ threshold. Looking carefully, we find that the ground-state of ${ }^{16} \mathrm{O}$ becomes unbound with respect to four- $\alpha$ decay starting at cutoff values $\Lambda \geqslant 550 \mathrm{MeV}$. However, without a comprehensive uncertainty analysis and the inclusion of higher-order particle-hole excitations in the $\mathrm{CC}$ method we cannot conclusively determine whether the SEP yields a stable ${ }^{16} \mathrm{O}$ ground state. We do note $\approx 5 \%$ increase in the binding energies when employing, e.g., the $\operatorname{CCSD}(\mathrm{T})$ approximation instead. This small shift makes ${ }^{16} \mathrm{O}$ bound with respect to four- $\alpha$ threshold throughout the cutoff range $\Lambda=450-600 \mathrm{MeV}$.

The NLO correction to the PC with perturbative $P$ waves is not fully known. It consists of at least the NLO ${ }^{1} S_{0}$ contribution in Eq. (4), which is what we employ here. In Ref. [53] it is proposed that the long-range OPE contribution at ${ }^{1} P_{1},{ }^{3} P_{1}$, and ${ }^{3} P_{2}-{ }^{3} F_{2}$ channels might belong to NLO as well, although the relative importance between the $\mathrm{NLO}^{1} S_{0}$ contribution and the long-range $P$-wave contributions is yet to be understood. Unfortunately, even with the less-repulsive choice-where only the NLO ${ }^{1} S_{0}$ contribution enters-the NLO shift is more or less always repulsive for $\Lambda=450-600 \mathrm{MeV}$, see Fig. 12 . Thus, it appears that the NLO correction to a PC with perturbative $P$ waves is unlikely to improve upon the results for ${ }^{16} \mathrm{O}$, at least within the cutoff range $\Lambda=450-550 \mathrm{MeV}$. In summary, the success of this PC also appears to be limited to lighter nuclei.

\section{SUMMARY AND IMPLICATIONS FOR FUTURE WORK}

In this work we have performed $a b$ initio NCSM and CC calculations of ${ }^{3} \mathrm{H},{ }^{3,4} \mathrm{He},{ }^{6} \mathrm{Li}$, and ${ }^{16} \mathrm{O}$ at $\mathrm{LO}$ and NLO in $\chi$ EFT using several PC schemes which all exhibit RG-invariance in the $\mathrm{NN}$ sector. We include all subleading corrections perturbatively. We employed the MWPC of Refs. [42-44], the dibaryon fields of Ref. [45], the separable PC of Ref. [79], and the recently proposed perturbative treatment of $P$ waves [53]. Our NCSM results indicate that the predictions of ground-state energies and point-proton radii in ${ }^{3} \mathrm{H}$ and ${ }^{3,4} \mathrm{He}$ exhibit signatures of RG invariance, and the converged values are mainly sensitive to variations in the two $S$-wave channels. Indeed, using different strategies for generating the amplitude in the ${ }^{1} S_{0}$ partial wave, the converged LO binding energies differ by $\approx 1 \mathrm{MeV}$ in ${ }^{3} \mathrm{H}$ and ${ }^{3} \mathrm{He}$, and $\approx 5 \mathrm{MeV}$ in ${ }^{4} \mathrm{He}$. In addition, for the $\mathrm{DBZ}$ approach we find a $\approx 20 \%$ variance due to the nonunique potential redefinition to handle the energy-dependent dibaryon structure.

The various RG-invariant NLO corrections to the groundstate energies for ${ }^{3} \mathrm{H}\left({ }^{4} \mathrm{He}\right)$ are as large as $\approx 2(\approx 7) \mathrm{MeV}$ with MWPC (attractive shifts, i.e., toward the experimental value). The corresponding shifts are smaller when using the SEP and DBZ potentials. In summary, all of the NLO results for $A=3,4$ nuclei are very reasonable and certainly agree with experiment at the level expected of NLO calculations, especially judging from the fact that no higher-body force has been added yet. Overall, the various PC schemes yield similar and realistic descriptions of $A \leqslant 4$ nuclei. 
However, this work has revealed a number of problems when applying the RG-invariant PC schemes to the study of nuclei with $A>4$. Some of the flaws of the MWPC interactions are seen already in the predictions of the ground-state energy and radius of ${ }^{6} \mathrm{Li}$. Contrary to experiment, the ground state of this nucleus was predicted slightly above the $\alpha+d$ threshold, even when considering the estimated uncertainties due to the IR extrapolation. Furthermore, we find too small radii and a strong cutoff dependence in the quadrupole moment. These results also motivate the detailed study of ${ }^{16} \mathrm{O}$.

Based on the results of $\mathrm{CC}$ predictions for the ground-state energy of ${ }^{16} \mathrm{O}$, we conclude that none of the PC schemes employed in this work appear to yield a realistic description of this nucleus. In fact, the most important observation is that none of the RG-invariant PCs in $\chi$ EFT successfully manages to generate a realistic and spherical ${ }^{16} \mathrm{O}$ ground state at $\mathrm{LO}$, which leaves small hope for a remedy from perturbative corrections. Model-space limitations hinder us from explicitly demonstrating renormalizability at large cutoffs for $A \gtrsim 6$. Still, our limited-cutoff results reveal fundamental flaws in several of the recently developed PC schemes, which is a significant and unexpected discovery. Of course, future analyses of RG-invariant schemes deserve a more careful and systematic parameter estimation of the LECs and adequate handling of the model discrepancy due to neglected higherorder diagrams in the $\chi$ EFT expansion.

In summary, it appears that the essential nuclear-binding mechanism fails in all present RG-invariant PC schemes for $\chi$ EFT. A remedy to this important finding will be critical for the utilization of PC schemes that employ a perturbative inclusion of subleading orders. We conclude that one (or several) of the following scenarios must be true:

(i) We have failed to capture a very fine-tuned process in the renormalization of the relevant LO LECs that is responsible for generating realistic ground states in ${ }^{16} \mathrm{O}$ and ${ }^{6} \mathrm{Li}$.

(ii) There is a scale critical to the physical description of finite-size nuclei, which is not captured by the contact terms at $\Lambda \rightarrow \infty$. This conceivable scale was discussed recently in Ref. [38] using pionless EFT, although the possible implications in $\chi$ EFT remain unclear.

(iii) Something else is missing in the LO interaction for describing ${ }^{16} \mathrm{O}$ and ${ }^{6} \mathrm{Li}$, and most likely other nuclei.

Naturally, we cannot rule out scenarios (i) and (ii), but we would like to speculate that it is quite possible that, due to an increasing relative importance of many-body forces in larger systems-as sketched also in Sec. 4.3 of Ref. [109]—a NNN force, such as the $\Delta$-full Fujita-Miyazawa NNN force [157] or the $\Delta$-less NNN force, must be promoted to LO in a $\chi$ EFT for many-nucleon systems. This would entail a nucleon-number-dependent PC which in turn, unfortunately, opens for the inclusion of four-body forces in larger-mass nuclei and nuclear matter. Such nucleon-number-dependent PC schemes will be explored in our future work.

\section{ACKNOWLEDGMENTS}

We thank U. van Kolck, B. Long, T. Papenbrock, J. Rotureau, M.S. Sanchez, G. Rupak and Y.-H. Song for useful discussions and suggestions. G.H. acknowledges the hospitality of Chalmers University of Technology where most of this work was carried out. This work was supported by the European Research Council (ERC) under the European Unions Horizon 2020 research and innovation programme (Grant agreement No. 758027), the Swedish Research Council (Swedish: Vetenskapsrådet) (Grant No. 2017-04234), the Office of Nuclear Physics, U.S. Department of Energy, under grants desc0018223 (NUCLEI SciDAC-4 collaboration) and by the Field Work Proposal ERKBP72 at Oak Ridge National Laboratory (ORNL). The computations were enabled by resources provided by the Swedish National Infrastructure for Computing (SNIC) at Chalmers Centre for Computational Science and Engineering (C3SE), the National Supercomputer Centre (NSC) partially funded by the Swedish Research Council, the Innovative and Novel Computational Impact on Theory and Experiment (INCITE) program. This research used resources of the Oak Ridge Leadership Computing Facility located at ORNL, which is supported by the Office of Science of the Department of Energy under Contract No. DE-AC05-00OR22725.
[1] S. Weinberg, Phys. A (Amsterdam, Neth.) 96, 327 (1979).

[2] S. Weinberg, Phys. Lett. B 251, 288 (1990).

[3] S. Weinberg, Nucl. Phys. B 363, 3 (1991).

[4] C. Ordóñez, L. Ray, and U. van Kolck, Phys. Rev. Lett. 72, 1982 (1994).

[5] C. Ordóñez, L. Ray, and U. van Kolck, Phys. Rev. C 53, 2086 (1996).

[6] E. Epelbaoum, W. Glöckle, and U.-G. Meißner, Nucl. Phys. A 637, 107 (1998).

[7] E. Epelbaum, W. Glöckle, and U.-G. Meißner, Nucl. Phys. A 671, 295 (2000).

[8] D. Entem and R. Machleidt, Phys. Lett. B 524, 93 (2002).

[9] D. R. Entem and R. Machleidt, Phys. Rev. C 66, 014002 (2002).
[10] E. Epelbaum, W. Glöckle, and U.-G. Meißner, Nucl. Phys. A 747, 362 (2005).

[11] E. Epelbaum, H. Krebs, and U. G. Meißner, Eur. Phys. J. A 51, 53 (2015).

[12] U. V. Kolck, Prog. Part. Nucl. Phys. 43, 337 (1999).

[13] P. F. Bedaque and U. V. Kolck, Annu. Rev. Nucl. Part. Sci. 52, 339 (2002).

[14] E. Epelbaum, H.-W. Hammer, and U.-G. Meißner, Rev. Mod. Phys. 81, 1773 (2009).

[15] H.-W. Hammer, S. König, and U. van Kolck, Rev. Mod. Phys. 92, 025004 (2020).

[16] W. H. Dickhoff and C. Barbieri, Prog. Part. Nucl. Phys. 52, 377 (2004).

[17] D. Lee, Prog. Part. Nucl. Phys. 63, 117 (2009). 
[18] S. K. Bogner, R. J. Furnstahl, and A. Schwenk, Prog. Part. Nucl. Phys. 65, 94 (2010).

[19] B. R. Barrett, P. Navrátil, and J. P. Vary, Prog. Part. Nucl. Phys. 69, 131 (2013).

[20] V. Somà, C. Barbieri, and T. Duguet, Phys. Rev. C 87, 011303(R) (2013).

[21] G. Hagen, T. Papenbrock, M. Hjorth-Jensen, and D. J. Dean, Rep. Prog. Phys. 77, 096302 (2014).

[22] H. Hergert, S. Bogner, T. Morris, A. Schwenk, and K. Tsukiyama, Phys. Rep. 621, 165 (2016).

[23] J. Carlson, S. Gandolfi, F. Pederiva, S. C. Pieper, R. Schiavilla, K. E. Schmidt, and R. B. Wiringa, Rev. Mod. Phys. 87, 1067 (2015).

[24] N. Barnea, W. Leidemann, and G. Orlandini, Nucl. Phys. A 650, 427 (1999).

[25] W. Glöckle, H. Witala, D. Hüber, H. Kamada, and J. Golak, Phys. Rep. 274, 107 (1996).

[26] T. R. Hemmert, B. R. Holstein, and J. Kambor, J. Phys. G 24, 1831 (1998).

[27] N. Kaiser, S. Gerstendorfer, and W. Weise, Nucl. Phys. A 637, 395 (1998).

[28] H. Krebs, E. Epelbaum, and U.-G. Meißner, Eur. Phys. J. A 32, 127 (2007).

[29] U. van Kolck, Nucl. Phys. A 645, 273 (1999).

[30] L. Platter, H.-W. Hammer, and U.-G. Meißner, Phys. Lett. B 607, 254 (2005).

[31] J. Kirscher, H. W. Grießhammer, D. Shukla, and H. M. Hofmann, Eur. Phys. J. A 44, 239 (2010).

[32] J. Kirscher, N. Barnea, D. Gazit, F. Pederiva, and U. van Kolck, Phys. Rev. C 92, 054002 (2015).

[33] V. Lensky, M. C. Birse, and N. R. Walet, Phys. Rev. C 94, 034003 (2016).

[34] N. Barnea, L. Contessi, D. Gazit, F. Pederiva, and U. van Kolck, Phys. Rev. Lett. 114, 052501 (2015).

[35] I. Stetcu, B. Barrett, and U. van Kolck, Phys. Lett. B 653, 358 (2007).

[36] L. Contessi, A. Lovato, F. Pederiva, A. Roggero, J. Kirscher, and U. van Kolck, Phys. Lett. B 772, 839 (2017).

[37] A. Bansal, S. Binder, A. Ekström, G. Hagen, G. R. Jansen, and T. Papenbrock, Phys. Rev. C 98, 054301 (2018).

[38] M. Schäfer, L. Contessi, J. Kirscher, and J. Mareš, Phys. Lett. B 816, 136194 (2021).

[39] J. Gasser and H. Leutwyler, Ann. Phys. (NY) 158, 142 (1984).

[40] V. Bernard, N. Kaiser, and U.-G. Meißner, Int. J. Mod. Phys. E 4, 193 (1995).

[41] A. Nogga, R. G. E. Timmermans, and U. van Kolck, Phys. Rev. C 72, 054006 (2005).

[42] B. Long and C.-J. Yang, Phys. Rev. C 84, 057001 (2011).

[43] B. Long and C.-J. Yang, Phys. Rev. C 85, 034002 (2012).

[44] B. Long and C.-J. Yang, Phys. Rev. C 86, 024001 (2012).

[45] M. S. Sanchez, C.-J. Yang, B. Long, and U. van Kolck, Phys. Rev. C 97. 024001 (2018).

[46] M. C. Birse, Phys. Rev. C 74, 014003 (2006).

[47] M. C. Birse, Phys. Rev. C 76, 034002 (2007).

[48] M. Birse, PoS(CD09), 078 (2010).

[49] M. Pavon Valderrama, Phys. Rev. C 83, 024003 (2011).

[50] M. Pavon Valderrama, Phys. Rev. C 84, 064002 (2011).

[51] B. Long, Phys. Rev. C 88, 014002 (2013).

[52] M. P. Valderrama, M. S. Sánchez, C.-J. Yang, B. Long, J. Carbonell, and U. van Kolck, Phys. Rev. C 95, 054001 (2017).

[53] S. Wu and B. Long, Phys. Rev. C 99, 024003 (2019).
[54] Y.-H. Song, R. Lazauskas, and U. V. Kolck, Phys. Rev. C 96, 024002 (2017).

[55] P. Navrátil, J. P. Vary, and B. R. Barrett, Phys. Rev. Lett. 84, 5728 (2000).

[56] P. Navrátil, J. P. Vary, and B. R. Barrett, Phys. Rev. C 62, 054311 (2000).

[57] F. Coester, Nucl. Phys. 7, 421 (1958).

[58] F. Coester and H. Kümmel, Nucl. Phys. 17, 477 (1960).

[59] J. Č́ížek, J. Chem. Phys. 45, 4256 (1966).

[60] J. Čížek, Adv. Chem. Phys., 14, 35 (1969).

[61] H. Kümmel, K. Lührmann, and J. Zabolitzky, Phys. Rep. 36, 1 (1978).

[62] R. J. Bartlett and M. Musiał, Rev. Mod. Phys. 79, 291 (2007).

[63] J. Simonis, S. R. Stroberg, K. Hebeler, J. D. Holt, and A. Schwenk, Phys. Rev. C 96, 014303 (2017).

[64] T. D. Morris, J. Simonis, S. R. Stroberg, C. Stumpf, G. Hagen, J. D. Holt, G. R. Jansen, T. Papenbrock, R. Roth, and A. Schwenk, Phys. Rev. Lett. 120, 152503 (2018).

[65] P. Gysbers, G. Hagen, J. D. Holt, G. R. Jansen, T. D. Morris, P. Navrátil, T. Papenbrock, S. Quaglioni, A. Schwenk, S. R. Stroberg, and K. A. Wendt, Nat. Phys. 15, 428 (2019).

[66] V. Somà, P. Navrátil, F. Raimondi, C. Barbieri, and T. Duguet, Phys. Rev. C 101, 014318 (2020).

[67] S. A. Coon, M. I. Avetian, M. K. G. Kruse, U. van Kolck, P. Maris, and J. P. Vary, Phys. Rev. C 86, 054002 (2012).

[68] R. J. Furnstahl, G. Hagen, and T. Papenbrock, Phys. Rev. C 86, 031301(R) (2012).

[69] S. N. More, A. Ekström, R. J. Furnstahl, G. Hagen, and T. Papenbrock, Phys. Rev. C 87, 044326 (2013).

[70] R. J. Furnstahl, G. Hagen, T. Papenbrock, and K. A. Wendt, J. Phys. G 42, 034032 (2015).

[71] K. A. Wendt, C. Forssén, T. Papenbrock, and D. Saaf, Phys. Rev. C 91, 061301(R) (2015).

[72] E. P. Wigner, Phys. Rev. 98, 145 (1955).

[73] D. R. Phillips and T. D. Cohen, Phys. Lett. B 390, 7 (1997).

[74] J. A. Oller and D. R. Entem, Ann. Phys. (NY) 411, 167965 (2019).

[75] J. A. Oller, Phys. Rev. C 93, 024002 (2016).

[76] D. R. Entem and J. A. Oller, Phys. Lett. B 773, 498 (2017).

[77] E. Epelbaum, A. M. Gasparyan, J. Gegelia, and U.-G. Meißner, Eur. Phys. J. A 54, 186 (2018).

[78] E. Epelbaum, A. M. Gasparyan, J. Gegelia, U.-G. Meißner, and X. L. Ren, Eur. Phys. J. A 56, 152 (2020).

[79] B. L. Shaowei Wu (private communication).

[80] G. Hagen, A. Ekström, C. Forssén, G. R. Jansen, W. Nazarewicz, T. Papenbrock, K. A. Wendt, S. Bacca, N. Barnea, B. Carlsson, C. Drischler, K. Hebeler, M. Hjorth-Jensen, M. Miorelli, G. Orlandini, A. Schwenk, and J. Simonis, Nat. Phys. 12, 186 (2015).

[81] S. Elhatisari, D. Lee, G. Rupak, E. Epelbaum, H. Krebs, T. A. Lähde, T. Luu, and U.-G. Meißner, Nature (London) 528, 111 (2015).

[82] C. Drischler, K. Hebeler, and A. Schwenk, Phys. Rev. Lett. 122, 042501 (2019).

[83] A. Nogga, Chiral Dynamics 2006 (World Scientific, 2007), pp. 182-193.

[84] A. Kievsky, S. Rosati, M. Viviani, L. E. Marcucci, and L. Girlanda, J. Phys. G 35, 063101 (2008). 
[85] P. Navrátil, V. G. Gueorguiev, J. P. Vary, W. E. Ormand, and A. Nogga, Phys. Rev. Lett. 99, 042501 (2007).

[86] S. Bacca, N. Barnea, W. Leidemann, and G. Orlandini, Phys. Rev. Lett. 102, 162501 (2009).

[87] A. Ekström, G. Baardsen, C. Forssén, G. Hagen, M. HjorthJensen, G. R. Jansen, R. Machleidt, W. Nazarewicz, T. Papenbrock, J. Sarich, and S. M. Wild, Phys. Rev. Lett. 110, 192502 (2013).

[88] A. Ekström, G. R. Jansen, K. A. Wendt, G. Hagen, T. Papenbrock, B. D. Carlsson, C. Forssén, M. Hjorth-Jensen, P. Navrátil, and W. Nazarewicz, Phys. Rev. C 91, 051301(R) (2015).

[89] A. Ekström, G. Hagen, T. D. Morris, T. Papenbrock, and P. D. Schwartz, Phys. Rev. C 97, 024332 (2018).

[90] W. G. Jiang, A. Ekström, C. Forssén, G. Hagen, G. R. Jansen, and T. Papenbrock, Phys. Rev. C 102, 054301 (2020).

[91] D. R. Entem, R. Machleidt, and Y. Nosyk, Phys. Rev. C 96, 024004 (2017).

[92] D. R. Entem, N. Kaiser, R. Machleidt, and Y. Nosyk, Phys. Rev. C 92, 064001 (2015).

[93] P. Reinert, H. Krebs, and E. Epelbaum, Eur. Phys. J. A 54, 86 (2018).

[94] R. Machleidt, Phys. Rev. C 63, 024001 (2001).

[95] R. B. Wiringa, V. G. J. Stoks, and R. Schiavilla, Phys. Rev. C 51, 38 (1995).

[96] V. G. J. Stoks, R. A. M. Klomp, M. C. M. Rentmeester, and J. J. de Swart, Phys. Rev. C 48, 792 (1993).

[97] C.-J. Yang, Ch. Elster, and D. R. Phillips, Phys. Rev. C 80, 034002 (2009).

[98] C.-J. Yang, Ch. Elster, and D. R. Phillips, Phys. Rev. C 80, 044002 (2009).

[99] C. Zeoli, R. Machleidt, and D. R. Entem, Few-Body Syst. 54, 2191 (2012).

[100] E. Epelbaum and J. Gegelia, Eur. Phys. J. A 41, 341 (2009).

[101] E. Epelbaum, J. Gegelia, and U.-G. Meißner, Nucl. Phys. B 925, 161 (2017).

[102] E. Epelbaum, J. Gegelia, and U.-G. Meißner, Commun. Theor. Phys. 69, 303 (2018).

[103] E. Epelbaum and U.-G. Meißner, Few-Body Syst. 54, 2175 (2013).

[104] L. Bingwei, Eur. Phys. J. A 25, 1641006 (2016).

[105] M. P. Valderrama, Eur. Phys. J. A 55, 55 (2019).

[106] M. P. Valderrama, arXiv:1902.08172.

[107] E. Epelbaum, A. M. Gasparyan, J. Gegelia, and U.-G. Meißner, Eur. Phys. J. A 55, 56 (2019).

[108] C. J. Yang and B. Long, in Chiral Symmetry in Hadrons and Nuclei, edited by L.-S. Geng, J. Meng, Q. Zhao, and B.-S. Zou (World Scientific, Singapore, 2014), p. 218.

[109] C. J. Yang, Eur. Phys. J. A 56, 96 (2020).

[110] U. van Kolck, Front. Phys. 8, 79 (2020).

[111] D. B. Kaplan, M. J. Savage, and M. B. Wise, Nucl. Phys. B 534, 329 (1998).

[112] D. B. Kaplan, M. J. Savage, and M. B. Wise, Nucl. Phys. B 478, 629 (1996).

[113] A. Gezerlis, I. Tews, E. Epelbaum, S. Gandolfi, K. Hebeler, A. Nogga, and A. Schwenk, Phys. Rev. Lett. 111, 032501 (2013).

[114] A. Gezerlis, I. Tews, E. Epelbaum, M. Freunek, S. Gandolfi, K. Hebeler, A. Nogga, and A. Schwenk, Phys. Rev. C 90, 054323 (2014).
[115] B.-N. Lu, N. Li, S. Elhatisari, D. Lee, E. Epelbaum, and U.-G. Meißner, Phys. Lett. B 797, 134863 (2019).

[116] A. Dyhdalo, R. J. Furnstahl, K. Hebeler, and I. Tews, Phys. Rev. C 94, 034001 (2016).

[117] S. Konig, H.-W. Griesshammer, H.-W. Hammer, and U. van Kolck, J. Phys. G 43, 055106 (2016).

[118] R. J. Furnstahl, N. Klco, D. R. Phillips, and S. Wesolowski, Phys. Rev. C 92, 024005 (2015).

[119] B. D. Carlsson, A. Ekström, C. Forssén, D. F. Strömberg, G. R. Jansen, O. Lilja, M. Lindby, B. A. Mattsson, and K. A. Wendt, Phys. Rev. X 6, 011019 (2016).

[120] S. Wesolowski, N. Klco, R. J. Furnstahl, D. R. Phillips, and A. Thapaliya, J. Phys. G 43, 074001 (2016).

[121] M. P. Valderrama and E. R. Arriola, Phys. Rev. C 72, 044007 (2005).

[122] P. Navrátil, G. P. Kamuntavičius, and B. R. Barrett, Phys. Rev. C 61, 044001 (2000).

[123] C. Forssén, B. D. Carlsson, H. T. Johansson, D. Saaf, A. Bansal, G. Hagen, and T. Papenbrock, Phys. Rev. C 97, 034328 (2018).

[124] Y.-H. Song, R. Lazauskas, and U. van Kolck, Phys. Rev. C 100, 019901 (2019).

[125] H. W. Grießhammer, PoS(CD15), 104 (2015).

[126] H. W. Grießhammer, Eur. Phys. J. A 56, 118 (2020).

[127] R. Furnstahl, D. Phillips, and S. Wesolowski, J. Phys. G 42, 034028 (2015).

[128] M. Schindler and D. Phillips, Ann. Phys. (NY) 324, 682 (2009).

[129] J. A. Melendez, S. Wesolowski, and R. J. Furnstahl, Phys. Rev. C 96, 024003 (2017).

[130] S. Wesolowski, R. Furnstahl, J. Melendez, and D. Phillips, J. Phys. G 46, 045102 (2019).

[131] J. A. Melendez, R. J. Furnstahl, D. R. Phillips, M. T. Pratola, and S. Wesolowski, Phys. Rev. C 100, 044001 (2019).

[132] J. L. Friar, Few-Body Syst. 22, 161 (1997).

[133] C.-J. Yang, Phys. Rev. C 94, 064004 (2016).

[134] S. Binder et al. (LENPIC), Phys. Rev. C 98, 014002 (2018).

[135] S. Binder, J. Langhammer, A. Calci, and R. Roth, Phys. Lett. B 736, 119 (2014).

[136] D. Logoteta, I. Bombaci, and A. Kievsky, Phys. Rev. C 94, 064001 (2016).

[137] D. R. Tilley, C. M. Cheves, J. L. Godwin, G. M. Hale, H. M. Hofmann, J. H. Kelley, C. G. Sheu, and H. R. Weller, Nucl. Phys. A 708, 3 (2002).

[138] C. Forssen, E. Caurier, and P. Navratil, Phys. Rev. C 79, 021303(R) (2009).

[139] T. Otsuka, T. Suzuki, J. D. Holt, A. Schwenk, and Y. Akaishi, Phys. Rev. Lett. 105, 032501 (2010).

[140] G. Hagen, M. Hjorth-Jensen, G. R. Jansen, R. Machleidt, and T. Papenbrock, Phys. Rev. Lett. 108, 242501 (2012).

[141] H. Hergert, S. Binder, A. Calci, J. Langhammer, and R. Roth, Phys. Rev. Lett. 110, 242501 (2013).

[142] A. Cipollone, C. Barbieri, and P. Navrátil, Phys. Rev. Lett. 111, 062501 (2013).

[143] E. Epelbaum, H. Krebs, T. A. Lähde, D. Lee, Ulf-G. Meißner, and G. Rupak, Phys. Rev. Lett. 112, 102501 (2014).

[144] V. Lapoux, V. Somà, C. Barbieri, H. Hergert, J. D. Holt, and S. R. Stroberg, Phys. Rev. Lett. 117, 052501 (2016).

[145] A. Ekström and G. Hagen, Phys. Rev. Lett. 123, 252501 (2019). 
[146] A. G. Taube and R. J. Bartlett, J. Chem. Phys. 128, 044110 (2008).

[147] G. Hagen, T. Papenbrock, D. J. Dean, and M. Hjorth-Jensen, Phys. Rev. C 82, 034330 (2010).

[148] T. Duguet and G. Hagen, Phys. Rev. C 85, 034330 (2012).

[149] M. G. Mayer, Phys. Rev. 75, 1969 (1949).

[150] S. J. Novario, G. Hagen, G. R. Jansen, and T. Papenbrock, Phys. Rev. C 102, 051303(R) (2020).

[151] D. B. Kaplan, Nucl. Phys. B 494, 471 (1997).
[152] B. H. McKellar and C. M. McKay, Aust. J. Phys. 36, 607 (1983).

[153] R. Yekken and R. J. Lombard, J. Phys. A: Math. Theor. 43, 125301 (2010).

[154] Y. Yamaguchi, Phys. Rev. 95, 1628 (1954).

[155] M. Sanchez Sanchez, N. A. Smirnova, A. M. Shirokov, P. Maris, and J. P. Vary, Phys. Rev. C 102, 024324 (2020).

[156] R. Peng, S. Lyu, and B. Long, Commun. Theor. Phys. 72, 095301 (2020).

[157] J. Fujita and H. Miyazawa, Prog. Theor. Phys. 17, 360 (1957). 